\title{
Implementation of 2D Discrete Wavelet Transform by Number Theoretic Transform and 2D Overlap-Save Method
}

\author{
Lina Yang, ${ }^{1,2}$ Yuan Yan Tang, ${ }^{1}$ and Qi Sun ${ }^{3}$ \\ ${ }^{1}$ Department of Computer and Information Science, University of Macau, Avenida Padre Tomas Pereira, Taipa 1356, Macau \\ ${ }^{2}$ Department of Mathematics and Computer Science, Guangxi Normal University of Nationalities, Chongzuo 532200, China \\ ${ }^{3}$ Department of Mathematics, Sichuan University, Chengdu, Sichuan 610064, China
}

Correspondence should be addressed to Yuan Yan Tang; yytang@umac.mo

Received 14 January 2014; Accepted 25 April 2014; Published 27 May 2014

Academic Editor: Cristian Toma

Copyright (C) 2014 Lina Yang et al. This is an open access article distributed under the Creative Commons Attribution License, which permits unrestricted use, distribution, and reproduction in any medium, provided the original work is properly cited.

\begin{abstract}
To reduce the computation complexity of wavelet transform, this paper presents a novel approach to be implemented. It consists of two key techniques: (1) fast number theoretic transform(FNTT) In the FNTT, linear convolution is replaced by the circular one. It can speed up the computation of 2D discrete wavelet transform. (2) In two-dimensional overlap-save method directly calculating the FNTT to the whole input sequence may meet two difficulties; namely, a big modulo obstructs the effective implementation of the FNTT and a long input sequence slows the computation of the FNTT down. To fight with such deficiencies, a new technique which is referred to as $2 \mathrm{D}$ overlap-save method is developed. Experiments have been conducted. The fast number theoretic transform and $2 \mathrm{D}$ overlap-method have been used to implement the dyadic wavelet transform and applied to contour extraction in pattern recognition.
\end{abstract}

\section{Introduction}

Wavelet transform plays an important role in image processing, pattern recognition, document analyses, and so forth [112]. The basic operation of it, in fact, is a linear convolution.

In digital signal processing, a signal is represented by a discrete sequence. Therefore, the discrete wavelet transform can be utilized to process it. We have [13]

$$
\begin{aligned}
W_{s}^{i} f(n, m) & =\iint f(u, v) \psi_{s}^{i}(n-u, m-v) d u d v \\
& =\sum_{k, l} f(n-1-k, m-1-l) \psi_{k, l}^{s, i},
\end{aligned}
$$

where

$$
\begin{aligned}
\psi_{k, l}^{s, i} & =\iint_{[k, k+1] \times[l, l+1]} \psi_{s}^{i}(u, v) d u d v \\
& =\int_{k / s}^{(k+1) / s} d u \int_{l / s}^{(l+1) / s} \psi^{i}(u, v) d v, \quad i=1,2 .
\end{aligned}
$$

Explicitly, either continuous wavelet transform or discrete wavelet transform is essentially the operation of filtering.
Directly calculating (1) may be time-consuming, because the number of multiplication operations may come large in this way. Although Mallat algorithm [14] can implement the wavelet transform successfully in some special cases, that is, in an orthogonal wavelet basis or in the multiresolution analysis, it is not a solution of all kinds of wavelet transforms. Therefore, studying how to speed up the general discrete wavelet transform is of great practical significance.

Actually, the filtering is a linear convolution in signal processing. In the following, without loss of generality, we will begin with the definition of Linear Convolution of two $2 \mathrm{D}$ sequences.

The equation for a $2 \mathrm{D}$ finite impulse response of a filter can be represented in the similar way and given by

$$
\begin{aligned}
y_{n_{1}, n_{2}} & =\sum_{\substack{k_{1}=0 \\
n_{1}-k_{1} \geq 0, n_{2}-k_{2} \geq 0}}^{l_{2}-1} \sum_{k_{2}=0}^{m_{2}-1} h_{k_{1}, k_{2}} x_{n_{1}-k_{1}, n_{2}-k_{2}} \\
n_{1} & =0,1, \ldots, \quad n_{2}=0,1, \ldots,
\end{aligned}
$$


where $x_{n_{1}, n_{2}}$ and $y_{n_{1}, n_{2}}\left(n_{1}=0,1, \ldots, n_{2}=0,1, \ldots,\right)$ stand for the input and output of the filter, respectively, and $h_{k_{1}, k_{2}}$ denote the filtering coefficients $\left(k_{1}=0,1, \ldots, l_{2}-1, k_{2} \stackrel{2}{=}\right.$ $\left.0,1, \ldots, m_{2}-1\right)$.

In many certain applications, we are required to compute only such an output sequence which is with a finite length in (3). Let $x_{n_{1}, n_{2}}$ be a 2D finite input of the filter with lengths $l_{1}$ and $m_{1}$. We compute the finite output as follows:

$$
\begin{gathered}
y_{n_{1}, n_{2}}=\sum_{\substack{k_{1}=0 \\
0 \leq n_{1}-k_{1}<l_{1} \\
0 \leq n_{2}-k_{2}<m_{1}}}^{l_{2}-1} \sum_{k_{1}, k_{2}}^{m_{2}-1} x_{n_{1}-k_{1}, n_{2}-k_{2}} \\
n_{1}=0,1, \ldots, l_{1}-1, \quad n_{2}=0,1, \ldots, m_{1}-1 .
\end{gathered}
$$

In practice, the difference between the length of the input signal and that of the filter is often large. If we calculate convolution (4) directly, a large number of multiplication operations will be executed. In the meantime, a series of calculations to treat zeros will be performed, if fast Fourier transform (FFT) is used to speed up the computation. Both of these two methods will be time-consuming to compute the linear convolution. To overcome this problem, in this paper we will present a novel approach which is referred to our previous work as number theoretic transform (NTT) [1517]. Also, we will prove that the computation of the linear convolution can be replaced by that of the cyclic convolution of two $2 \mathrm{D}$ sequences with lengths $N_{1}=l_{1}+l_{2}-1$ and $N_{2}=m_{1}+m_{2}-1$, respectively.

For two $2 \mathrm{D}$ sequences $x_{n_{1}, n_{2}}$ and $h_{n_{1}, n_{2}}$ of finite lengths $N_{1}$ and $N_{2}$, their cyclic convolution can be written by

$$
\begin{array}{r}
y_{n_{1}, n_{2}}=\sum_{k_{1}=0}^{N_{1}-1} \sum_{k_{2}=0}^{N_{2}-1} x_{k_{1}, k_{2}} h_{\left\langle n_{1}-k_{1}\right\rangle_{N_{1}},\left\langle n_{2}-k_{2}\right\rangle_{N_{2}}} \\
=\sum_{k_{1}=0}^{N_{1}-1} \sum_{k_{2}=0}^{N_{2}-1} h_{k_{1}, k_{2}} x_{\left\langle n_{1}-k_{1}\right\rangle_{N_{1}},\left\langle n_{2}-k_{2}\right\rangle_{N_{2}}} \\
n_{1}=0,1, \ldots, N_{1}-1, \quad n_{2}=0,1, \ldots, N_{2}-1,
\end{array}
$$

where $\langle x\rangle_{N}$ denotes the remainder $r$ of $x$ modulo $N$; that is, $x=q N+r, q$ is any integer and $0 \leq r<N$.

The number theoretic transform (NTT) provides an effective way to calculate the cyclic convolution. However, there exist two difficulties, if directly applying the NTT to the whole input sequence, namely:

(i) a considerable big modulo $M$ has to be imposed. It obstructs the effective implementation of the NTT due to the limited length of the word which is used to store data in computers. It will, thus, become a bottleneck in the computation of convolution;

(iii) the advantage of the fast computation of the NTT cannot be reached when the difference between the length of the input sequence and that of the filter sequence is large. Thus, it may not speed up the calculation of convolution in this case.
To avoid using a big modulo $M$, we can use Chinese remainder theorem to reduce the length of the modulo $M$ and successfully apply the NTT to calculate the convolution. Unfortunately, the number of multiplication operations will be at least doubled, and thus the computational complexity will increase. The second key technique will be worked out in this paper to overcome the above difficulties and speed up the calculation of the convolution. It is termed 2D overlapsave method, which is an expansion of the 1D overlapsave method. It can be used to implement the $2 \mathrm{D}$ wavelet transform when a big difference between the length of the input sequence $x_{n_{1}, n_{2}}$ and that of the filter sequence $h_{n_{1}, n_{2}}$ occurs. This method consists of three steps.

(i) First, the original 2D input sequence is equivalently divided into many small separated sections. This division brings two evident improvements.

(a) The size of each section is much smaller than that of the whole input sequence, such that a small modulo $M$ can be used, and the NTT can, therefore, be performed in a computer system.

(b) The difference between the length of one section and that of the filter sequence becomes smaller, which makes the effective application of the NTT.

(ii) In the second step, we calculate the cyclic convolution of each section with the filter sequence by NTT.

(iii) Finally, the result of (4) is obtained by picking out data from each of the results calculated in the second step and combining these data together.

In comparison with direct method and fast Fourier transform (FFT), it will be proved explicitly that the number of multiplication operations in the $2 \mathrm{D}$ overlap-save method will be smaller than that in any of those two methods in many particular cases.

In the next section, the number theoretic transform (NTT) will be presented. Details of the 2D overlap-method will be discussed in Section 3. A comparison of computation of the multiplication in three methods will be given in Section 4. A computational example will be presented as well as a practical experiment in Section 5 which will verify the efficiency of the proposed approach. In the experiment, the fast number theoretic transform and 2D overlap-method were applied to implement the dyadic wavelet transform extracting the contours in recognition of Chinese handwriting.

\section{Number Theoretic Transform (NTT)}

Suppose that $h_{k_{1}, k_{2}}$ are the filter coefficients $\left(k_{1}=0,1, \ldots, l_{2}-\right.$ $\left.1, k_{2}=0,1, \ldots, m_{2}-1\right) ; x_{n_{1}, n_{2}}$ and $y_{n_{1}, n_{2}}\left(n_{1}=0,1, \ldots, l_{1}-\right.$ $\left.1, n_{2}=0,1, \ldots, m_{1}-1\right)$ are the input and output of the filter, respectively. In the concrete applications of the discrete wavelet transform (DWT), the lengths of the input and output are very long ( $l_{1}$ and $m_{1}$ are very big), while lengths of the filter coefficients are short $\left(l_{2}\right.$ and $m_{2}$ are small). Therefore, if 
the direct method is used to calculate their linear convolution, the number of multiplication operations will be large. In this section, the 2D number theoretic transform (FNTT) will be applied to calculate the $2 \mathrm{D}$ circular convolution of $2 \mathrm{D}$ sequences of lengths $N_{1}=l_{1}+l_{2}-1$ and $N_{2}=m_{1}+m_{2}-1$. In a $2 \mathrm{D}$ number theoretic transform (NTT), if a modulo $M$ is too big, the Chinese remainder theorem (CRT) will be utilized to reduce the length.

2.1. 2D Linear Convolution and 2D Circular Convolution. Given two 2-dimensional sequences $x_{n_{1}, n_{2}}\left(n_{1}=0,1, \ldots, l_{1}-\right.$ $\left.1, n_{2}=0,1, \ldots, m_{1}-1\right)$ and $h_{n_{1}, n_{2}}\left(n_{1}=0,1, \ldots, l_{2}-1, n_{2}=\right.$ $\left.0,1, \ldots, m_{2}-1\right)$, their linear convolution is defined by

$$
\begin{gathered}
y_{n_{1}, n_{2}}=\sum_{\substack{k_{1}=0 \\
0 \leq n_{1}-k_{1}<l_{2} \\
0 \leq n_{2}-k_{2}<m_{2}}}^{l_{1}-1} \sum_{k_{1}, k_{2}}^{m_{1}-1} h_{n_{1}-k_{1}, n_{2}-k_{2}} \\
=\sum_{k_{1}=0}^{l_{2}-1} \sum_{k_{2}=0}^{m_{2}-1} h_{k_{1}, k_{2}} x_{n_{1}-k_{1}, n_{2}-k_{2}} \\
0 \leq n_{1}-k_{1}<l_{1} \\
n_{1}=0,1, \ldots, l_{1}+l_{1}-2, \quad l_{2}=0,1, \ldots, m_{1}+m_{2}-2 .
\end{gathered}
$$

Given two 2-dimensional sequences $x_{n_{1}, n_{2}}$ and $h_{n_{1}, n_{2}}$ of lengths $N_{1}$ and $N_{2}$, respectively, their circular convolution is

$$
\begin{array}{r}
y_{n_{1}, n_{2}}=\sum_{k_{1}=0}^{N_{1}-1} \sum_{k_{2}=0}^{N_{2}-1} x_{k_{1}, k_{2}} h_{\left\langle n_{1}-k_{1}\right\rangle_{N_{1}},\left\langle n_{2}-k_{2}\right\rangle_{N_{2}}} \\
=\sum_{k_{1}=0}^{N_{1}-1} \sum_{k_{2}=0}^{N_{2}-1} h_{k_{1}, k_{2}} x_{\left\langle n_{1}-k_{1}\right\rangle_{N_{1}},\left\langle n_{2}-k_{2}\right\rangle_{N_{2}}} \\
n_{1}=0,1, \ldots, N_{1}-1, \quad n_{2}=0,1, \ldots, N_{2}-1,
\end{array}
$$

where $\langle x\rangle_{N}$ denotes the remainder $r, x=q N+r, 0 \leq r<N$.

Proposition 1. The linear convolution (6) can be computed by computing circular convolution of two 2-dimensional sequences of lengths $N_{1}=l_{1}+l_{2}-1$ and $N_{2}=m_{1}+m_{2}-1$, respectively.

2.2. Using 2D Number Theoretic Transform to Calculate the $2 D$ Circular Convolution. Let $M$ be a positive integer and suppose that $x_{n_{1}, n_{2}}$ and $h_{n_{1}, n_{2}}$ are two 2-dimensional integer sequences $\left(n_{1}=0,1, \ldots, N_{1}-1, n_{2}=0,1, \ldots, N_{2}-1\right)$.

Let

$$
\begin{aligned}
X_{k_{1}, k_{2}} & \equiv \sum_{n_{1}=0}^{N_{1}-1} \sum_{n_{2}=0}^{N_{2}-1} x_{n_{1}, n_{2}} \alpha^{n_{1} k_{1}} \beta^{n_{2} k_{2}}(\bmod M), \\
H_{k_{1}, k_{2}} & \equiv \sum_{n_{1}=0}^{N_{1}-1} \sum_{n_{2}=0}^{N_{2}-1} h_{n_{1}, n_{2}} \alpha^{n_{1} k_{1}} \beta^{n_{2} k_{2}}(\bmod M), \\
Y_{k_{1}, k_{2}} & \equiv \sum_{n_{1}=0}^{N_{1}-1} \sum_{n_{2}=0}^{N_{2}-1} y_{n_{1}, n_{2}} \alpha^{n_{1} k_{1}} \beta^{n_{2} k_{2}}(\bmod M),
\end{aligned}
$$

where $\left(k_{1}=0,1, \ldots, N_{1}-1, k_{2}=0,1, \ldots, N_{2}-1\right)$, and $\alpha$ and $\beta$ are two given integers, and $y_{n_{1}, n_{2}}$ denote the circular convolution as shown in (7), $n_{1}=0,1, \ldots, N_{1}-1, n_{2}=$ $0,1, \ldots, N_{2}-1$.

If a 2-dimensional transform (8) has an inverse transform

$$
x_{n_{1}, n_{2}} \equiv N_{1}^{-1} \cdot N_{2}^{-1} \sum_{k_{1}=0}^{N_{1}-1} \sum_{k_{2}=0}^{N_{2}-1} X_{k_{1}, k_{2}} \alpha^{-n_{1} k_{1}} \alpha^{-n_{2} k_{2}}(\bmod M)
$$

$\left(n_{1}=0,1, \ldots, N_{1}-1, n_{2}=0,1, \ldots, N_{2}-1\right)$ and has circular convolution property,

$$
\begin{gathered}
Y_{k_{1}, k_{2}} \equiv X_{k_{1}, k_{2}} H_{k_{1}, k_{2}}(\bmod M) \\
\left(k_{1}=0,1, \ldots, N_{1}-1, k_{2}=0,1, \ldots, N_{2}-1\right),
\end{gathered}
$$

then congruence equation (8) is referred to as a 2dimensional number theoretic transform $\bmod M$ and can be abbreviated to NTT $\bmod M$.

Agarwal and Burrus gave some sufficient conditions for existence of 2-dimensional NTT given with $M>1, N_{1}>1$, and $N_{2}>1$, (see [15]). In [17], the authors gave some sufficient and necessary conditions for existence. We have the following theorem.

Theorem 2. If $M=p_{1}^{l_{1}} \cdots p_{s}^{l_{s}},\left(l_{j} \geq 1, j=1, \ldots, s\right), p_{1}, \ldots, p_{s}$ are distinct primes, then the congruence equation (8) is a 2dimensional NTT $\bmod M$ with the first dimension of length $N_{1}$ and the second dimension of length $N_{2}$, if and only if $\alpha^{N_{1}} \equiv$ $1(\bmod M), \beta^{N_{2}} \equiv 1(\bmod M)$ and $\alpha^{j} \not \equiv 1\left(\bmod p_{k}\right)(1 \leq$ $\left.j \leq N_{1}-1\right), \beta^{i} \neq \equiv 1\left(\bmod p_{k}\right)\left(1 \leq i \leq N_{2}-1\right), k=1, \ldots, s$.

Corollary 3. The congruence equation (8) is a 2-dimensional $N T T \bmod M$ if and only if

$$
\operatorname{lcm}\left[N_{1}, N_{2}\right] \mid \operatorname{gcd}\left(p_{1}-1, \ldots, p_{s}-1\right),
$$

where $\operatorname{lcm}\left[N_{1}, N_{2}\right]$ denotes the least common multiple of two integers $N_{1}$ and $N_{2}$ and $\operatorname{gcd}(a, b)$ denotes the greatest common divisor of two integers $a$ and $b$.

Corollary 4. Let $p$ be an odd prime, and $M=p$. Then the congruence equation (8) is a 2-dimensional NTT $\bmod p$, if and only if $\alpha^{N_{1}} \equiv 1(\bmod p)$ and $\alpha^{j} \neq \equiv 1(\bmod p)(1 \leq j \leq$ $\left.N_{1}-1\right), \beta^{N_{2}} \equiv 1(\bmod p)$, and $\beta^{j} \neq \equiv 1(\bmod p)(1 \leq j \leq$ $\left.N_{2}-1\right)$.

Clearly, from congruence equations (8), (9), (11), and (12), we can use the 2-dimensional NTT to calculate a 2dimensional circular convolution.

Finally, we obtain

$$
\begin{array}{r}
y_{n_{1}, n_{2}} \equiv \sum_{k_{1}=0}^{N_{1}-1} \sum_{k_{2}=0}^{N_{2}-1} x_{k_{1}, k_{2}} h_{\left\langle n_{1}-k_{1}\right\rangle_{N_{1}},\left\langle n_{2}-k_{2}\right\rangle_{N_{2}}}(\bmod M) \\
n_{1}=0,1, \ldots, N_{1}-1, n_{2}=0,1, \ldots, N_{2}-1 .
\end{array}
$$


Select $M$ so that

$$
\begin{aligned}
\left|y_{n_{1}, n_{2}}\right| \leq \min & \left\{\left.\left|x_{n_{1}, n_{2}}\right|\right|_{\max } \sum_{k_{1}=0}^{N_{1}-1} \sum_{k_{2}=0}^{N_{2}-1}\left|h_{k_{1}, k_{2}}\right|,\right. \\
& \left.\left|h_{n_{1}, n_{2}}\right|_{\max } \sum_{k_{1}=0}^{N_{1}-1} \sum_{k_{2}=0}^{N_{2}-1}\left|x_{k_{1}, k_{2}}\right|\right\}<\frac{M}{2},
\end{aligned}
$$

where $n_{1}=0,1, \ldots, N_{1}-1, n_{2}=0,1, \ldots, N_{2}-1$, and $M$ is an odd.

If we use the 2-dimensional NTT to calculate the 2dimensional circular convolution $y_{n_{1}, n_{2}}$ so that $\left|y_{n_{1}, n_{2}}\right|<M / 2$, then form congruence equation (14) we deduce that

$$
\begin{array}{r}
y_{n_{1}, n_{2}}=\sum_{k_{1}=0}^{N_{1}-1} \sum_{k_{2}=0}^{N_{2}-1} x_{k_{1}, k_{2}} h_{\left\langle n_{1}-k_{1}\right\rangle_{N_{1}}}\left\langle n_{2}-k_{2}\right\rangle_{N_{2}}, \\
n_{1}=0,1, \ldots, N_{1}-1, \quad n_{2}=0,1, \ldots, N_{2}-1 .
\end{array}
$$

2.3. Fast Number Theoretic Transform (FNTT). The idea of FFT can be used to perform the NTT. In this subsection, a theoretic description will be presented briefly. More details will be given in Section 5 .

Let

$$
\begin{array}{r}
U_{0, k_{2}} \equiv \sum_{n_{2}=0}^{N_{2}-1} x_{0, n_{2}} \beta^{n_{2} k_{2}}, \ldots, U_{N_{1}-1, k_{2}} \\
\equiv \sum_{n_{2}=0}^{N_{2}-1} x_{N_{1}-1, n_{2}} \beta^{n_{2} k_{2}}(\bmod M), \\
k_{2}=0,1, \ldots, N_{2}-1 .
\end{array}
$$

From congruence equation (17), we deduce that

$$
\begin{array}{r}
X_{k_{1}, 0} \equiv \sum_{n_{1}=0}^{N_{1}-1} U_{n_{1}, 0} \alpha^{n_{1} k_{1}}, \ldots, X_{k_{1}, N_{2}-1} \\
\equiv \sum_{n_{1}=0}^{N_{1}-1} U_{n_{1}, N_{2}-1} \alpha^{n_{1} k_{1}}(\bmod M), \\
k_{1}=0,1, \ldots, N_{1}-1 .
\end{array}
$$

Suppose that $N_{1}$ and $N_{2}$ satisfy inequalities, and we then can use the idea of FFT to calculate the congruence equations (17) and (18).

For computing every

$$
\begin{array}{r}
U_{n_{1}, k_{2}} \equiv \sum_{n_{2}=0}^{N_{2}-1} x_{n_{1}, n_{2}} \beta^{n_{2} k_{2}}(\bmod M), \\
\left(k_{2}=0,1, \ldots, N_{2}-1\right)
\end{array}
$$

in congruence equation (17), the numbers of all multiplication necessary are $\left(N_{2} \log N_{2}\right) / 2$ by using FFT algorithm. Hence, if using FFT algorithm to calculate the congruence

$$
\begin{array}{r}
U_{n_{1}, k_{2}} \equiv \sum_{n_{2}=0}^{N_{2}-1} x_{n_{1}, n_{2}} \beta^{n_{2} k_{2}}(\bmod M), \\
\left(k_{2}=0,1, \ldots, N_{2}-1\right)
\end{array}
$$

in congruence equation (17), the numbers of all multiplication necessary are $\left(\left(N_{1} N_{2}\right) / 2\right) \log N_{2}$.

Similarly, if using FFT algorithm to calculate all congruence equations in (18), then the numbers of all multiplication necessary are $\left(\left(N_{2} N_{1}\right) / 2\right) \log N_{1}$.

Therefore, if using the fast number theoretic transform (FNTT) to calculate the congruence equation (8), the number of all multiplication necessary is

$$
\frac{N_{1} N_{2}}{2} \log N_{2}+\frac{N_{2} N_{1}}{2} \log N_{1}=\frac{N_{1} N_{2}}{2} \log \left(N_{1} N_{2}\right) .
$$

If $M$ is very large, we may reduce the length of a word by using Chinese remainder theorem and it can be abbreviated to CRT. Therefore, we have the following proposition.

Proposition 5. Suppose that congruence equation (8) is a 2dimensional NTT $\bmod M$, where $M=p_{1}^{l_{1}} \cdots p_{s}^{l_{s}}, p_{1}, \ldots, p_{s}$ are distinct primes; then, we have a total of $s$ 2-dimensional NTT $\bmod p_{i}^{l_{i}}$, and they are described as follows:

$$
\begin{aligned}
& X_{k_{1}, k_{2}} \equiv \sum_{n_{1}=0}^{N_{1}-1} \sum_{n_{2}=0}^{N_{2}-1} x_{n_{1}, n_{2}} \alpha_{i}^{n_{1} k_{1}} \beta_{i}^{n_{2} k_{2}},\left(\bmod p_{i}^{l_{i}}\right) \\
& k_{1}=0,1, \ldots, N_{1}-1, \quad k_{2}=0,1, \ldots, N_{2}-1,
\end{aligned}
$$

$$
\begin{aligned}
& \text { where } \alpha_{i}=\langle\alpha\rangle_{p_{i}^{l_{i}}}, \beta_{i}=\langle\beta\rangle_{p_{i}^{i}}, i=1,2, \ldots, s . \\
& \text { If } \\
& y_{n_{1}, n_{2}}=\sum_{k_{1}=0}^{N_{1}-1} \sum_{k_{2}=0}^{N_{2}-1} x_{k_{1}, k_{2}} h_{\left\langle n_{1}-k_{1}\right\rangle_{N_{1}},\left\langle n_{2}-k_{2}\right\rangle_{N_{2}}} \equiv y_{n_{1}, n_{2}}^{(i)}\left(\bmod p_{i}^{l_{i}}\right) \\
& \quad\left(n_{1}=0,1, \ldots, N_{1}-1, n_{2}=0,1, \ldots, N_{2}-1\right),
\end{aligned}
$$

then

$$
\begin{gathered}
y_{n_{1}, n_{2}} \equiv \sum_{i=1}^{s} M_{i}^{\prime} M_{i} y_{n_{1}, n_{2}}^{(i)}(\bmod M) \\
\left(n_{1}=0,1, \ldots, N_{1}-1, n_{2}=0,1, \ldots, N_{2}-1\right),
\end{gathered}
$$

where $M=p_{i}^{l_{i}} M_{i}, M_{i}^{\prime} M_{i} \equiv 1\left(\bmod p_{i}^{l_{i}}\right), i=1, \ldots, s$. 
Proof. Because the congruence equation (8) is a 2-dimensional NTT $\bmod M$, hence

$$
\begin{aligned}
& \alpha^{N_{1}} \equiv 1(\bmod M), \quad \beta^{N_{2}} \equiv 1(\bmod M), \\
& \alpha^{N_{1}} \equiv 1\left(\bmod p_{i}\right), \quad \alpha^{j} \not \equiv 1\left(\bmod p_{i}\right), \quad 1 \leq j<N_{1} \text {, } \\
& \beta^{N_{2}} \equiv 1\left(\bmod p_{i}\right), \quad \beta^{h} \neq \equiv\left(\bmod p_{i}\right), \quad 1 \leq h<N_{2}, \\
& i=1,2, \ldots, s \text {, }
\end{aligned}
$$

so

$$
\begin{aligned}
& \alpha^{N_{1}} \equiv 1\left(\bmod p_{i}^{l_{i}}\right), \quad \beta^{N_{2}} \equiv 1\left(\bmod p_{i}^{l_{i}}\right), \\
& \alpha_{i}^{N_{1}} \equiv \alpha^{N_{1}} \equiv 1\left(\bmod p_{i}\right), \quad \alpha_{i}^{j} \not \equiv 1\left(\bmod p_{i}\right) \text {, } \\
& 1 \leq j<N_{1} \text {, } \\
& \beta_{i}^{N_{2}} \equiv \beta^{N_{2}} \equiv 1\left(\bmod p_{i}\right), \quad \beta_{i}^{h} \not \equiv 1\left(\bmod p_{i}\right), \\
& 1 \leq h<N_{2} \text {, } \\
& i=1, \ldots, s \text {. }
\end{aligned}
$$

Hence, the congruence equation (22) has a total of $s 2$ dimensional NTTs $\bmod p_{i}^{l_{i}}, i=1, \ldots, s$. From CRT and the congruence equation (23), we deduce that the congruence equation (24) holds.

\section{Two-Dimensional Overlap-Save Method}

Select two positive integers $N^{\prime}$ and $M^{\prime}$, so that $N^{\prime}+l_{2}-1=$ $N<l_{1}$ and $M^{\prime}+m_{2}-1=M<m_{1}$; either $N$ or $M$ is given by an integer power of 2 ; that is, $N=2^{d_{1}}$ and $M=2^{d_{2}}$ where $d_{1}$ and $d_{2}$ are two positive integers.

Assuming that the input sequence $x_{n_{1}, n_{2}}\left(n_{1}=\right.$ $\left.0,1, \ldots, l_{1}-1, n_{2}=0,1, \ldots, m_{1}-1\right)$ is divided into many small sections, which are termed submatrices,

$$
\begin{gathered}
\left\{x_{n_{1}+v_{1} N^{\prime}, n_{2}+v_{2} M^{\prime}}\right\}_{n_{1}=0, n_{2}=0}^{n_{1}=N-1, n_{2}=M-1} \\
\left(v_{1}=0,1, \ldots, v_{2}=0,1, \ldots\right) .
\end{gathered}
$$

The consecutive matrix is overlapped by the previous one. As a simple example, four blocks chosen from the divided input matrices are overlapped and are graphically shown in Figure 1. Parameters $v_{1}$ and $v_{2}$ can be considered as shift parameters, because changing either $v_{1}$ or $v_{2}$ will select different submatrix. For the first submatrix shown in Figure 1(a), we select $v_{1}=0$ and $v_{2}=0$. Second submatrix $\left(v_{1}=0\right.$ and $v_{2}=1$ ) is displayed in Figure 1(b). Third one with parameters of $v_{1}=1$ and $v_{2}=0$ is given in Figure $1(\mathrm{c})$. Fourth matrix shown in Figure $1(\mathrm{~d})$ has parameters $v_{1}=1$ and $v_{2}=1$. The procedure of the overlapping is also graphically illustrated in Figure 1.

For the operation of circular convolution, it is necessary to have two sequences with the same length. Taking (7) for example, the sequences $x_{n_{1}, n_{2}}$ and $h_{n_{1}, n_{2}}$ are of the same length of $N_{1} \times N_{2}$. Since the input sequence has already divided into submatrices of $N \times M$ by (27), the filter sequence has to be of length $N \times M$. Therefore, $N-l_{2}$ augmenting zeros are required to be added to the row and $M-m_{2}$ zeros to the column of the filter sequence $h_{n_{1}, n_{2}}$. As a result, the sequence $h_{n_{1}, n_{2}}$ becomes

$$
h_{n_{1}, n_{2}}^{\prime}= \begin{cases}h_{n_{1}, n_{2}} & \text { if } n_{1}=0,1, \ldots, l_{2}-1, n_{2}=0,1, \ldots, m_{2}-1 \\ 0 & \text { if } l_{2} \leq n_{1}<N \text { or } m_{2} \leq n_{2}<M,\end{cases}
$$

where $n_{1}=0,1, \ldots, N-1$ and $n_{2}=0,1, \ldots, M-1$.

The following form shows how a sequence $h_{n_{1}, n_{2}}^{\prime}$ is yielded by adding augmenting zeros to the filter sequence $h_{n_{1}, n_{2}}$ :

$$
\{h\} \Longrightarrow\left\{h^{\prime}\right\}=\left\{\begin{array}{ll}
\{h\} & \\
& 0
\end{array}\right\} \text {. }
$$

For each of the divided submatrices, we can give $v_{1}$ and $v_{2}$ and compute the circular convolution of two $2 \mathrm{D}$ sequences $x_{n_{1}+v_{1} N^{\prime}, n_{2}+v_{2} M^{\prime}}$ and $h_{n_{1}, n_{2}}^{\prime}\left(n_{1}=0,1, \ldots, N-1, n_{2}=\right.$ $0,1, \ldots, M-1)$ by

$$
\begin{aligned}
\bar{y}_{t_{1}, t_{2}} & =\sum_{n_{1}=0}^{N-1} \sum_{n_{2}=0}^{M-1} h_{n_{1}, n_{2}}^{\prime} x_{\left\langle t_{1}-n_{1}\right\rangle_{N}+v_{1} N^{\prime},\left\langle t_{2}-n_{2}\right\rangle_{M}+v_{2} M^{\prime}} \\
t_{1} & =0,1, \ldots, N-1, \quad t_{2}=0,1, \ldots, M-1 .
\end{aligned}
$$

From (28), we deduce that

$$
\bar{y}_{t_{1}, t_{2}}=\sum_{n_{1}=0}^{l_{2}-1} \sum_{n_{2}=0}^{m_{2}-1} h_{n_{1}, n_{2}} x_{\left\langle t_{1}-n_{1}\right\rangle_{N}+v_{1} N^{\prime},\left\langle t_{2}-n_{2}\right\rangle_{M}+v_{2} M^{\prime}}
$$

The result of (31) is that of circular convolution. As we will see below, there exists an important connection between the circular convolution and the linear one. In fact, since $n_{1}=$ $0,1, \ldots, l_{2}-1$ and $n_{2}=0,1, \ldots, m_{2}-1$, we can choose $t_{1}=$ $l_{2}-1, \ldots, N-1$ and $t_{2}=m_{2}-1, \ldots, M-1$ in (31); this makes $N>t_{1}-n_{1}>0$ and $M>t_{2}-n_{2}>0$ in (31). Thus, the remainder can exactly be equal to $t_{1}-n_{1}$ and $t_{2}-n_{2}$. Then, the circular convolution of (31) becomes

$$
\begin{aligned}
\bar{y}_{t_{1}, t_{2}} & =\sum_{n_{1}=0}^{l_{2}-1} \sum_{n_{2}=0}^{m_{2}-1} h_{n_{1}, n_{2}} x_{\left\langle t_{1}-n_{1}\right\rangle_{N}+v_{1} N^{\prime},\left\langle t_{2}-n_{2}\right\rangle_{M}+v_{2} M^{\prime}} \\
& =\sum_{n_{1}=0}^{l_{2}-1} \sum_{n_{2}=0}^{m_{2}-1} h_{n_{1}, n_{2}} x_{t_{1}-n_{1}+v_{1} N^{\prime}, t_{2}-n_{2}+v_{2} M^{\prime}} \\
& =y_{t_{1}+v_{1} N^{\prime}, t_{2}+v_{2} M^{\prime}} .
\end{aligned}
$$

In order to clearly understand the procedure of obtaining the components of linear convolution from the circular convolution equation (31), a graphic description of it can be found in Figure 2. We show a dashed rectangle with size of $N$ by $M$, in Figure 2(a), to represent the circular convolution which is described in (31) and a darkened rectangle with size of $N^{\prime}$ by $M^{\prime}$, to indicate the linear convolution stated in (32). 


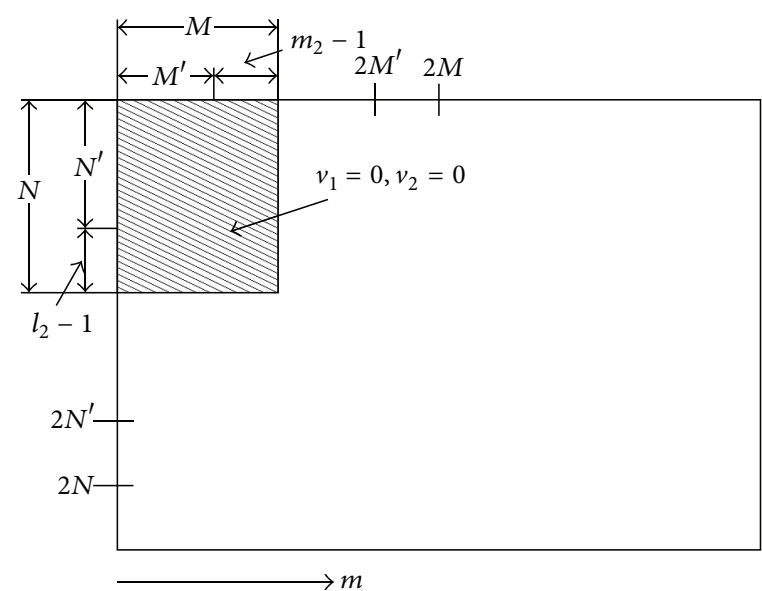

(a)

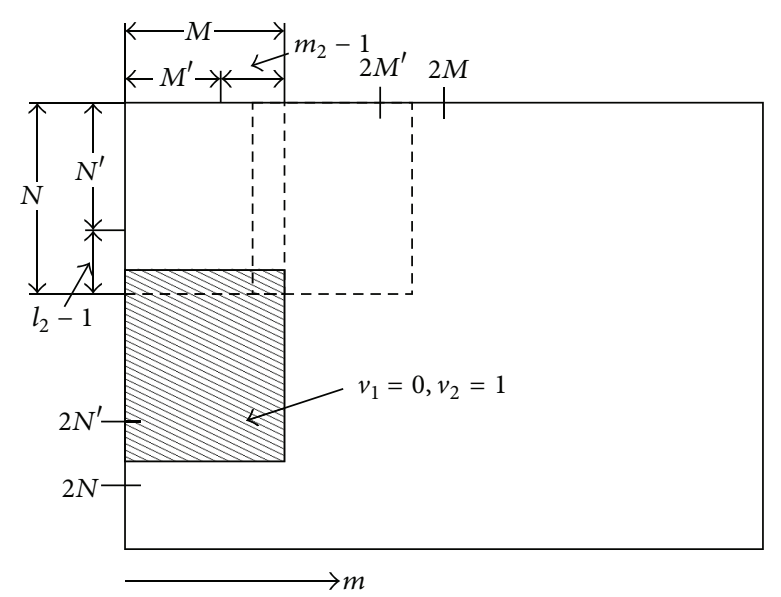

(c)

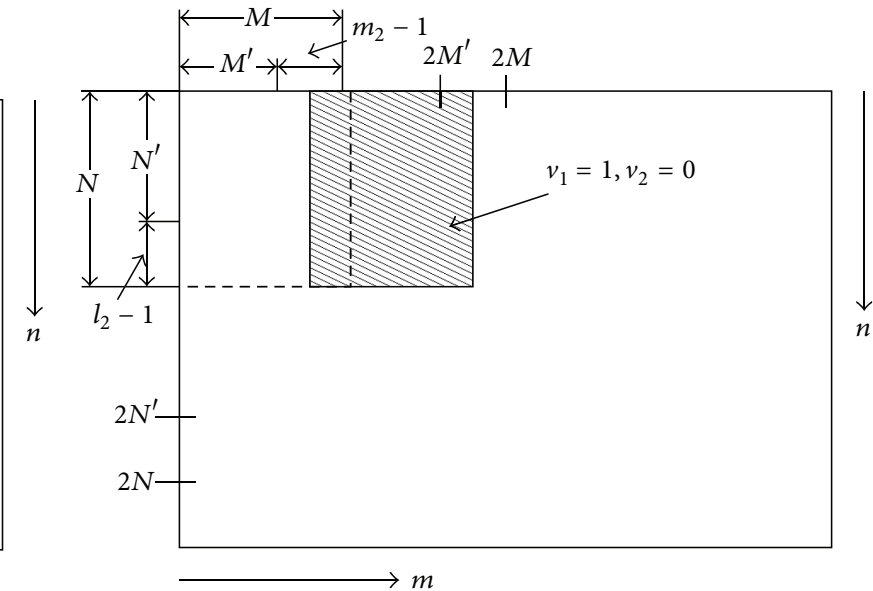

(b)

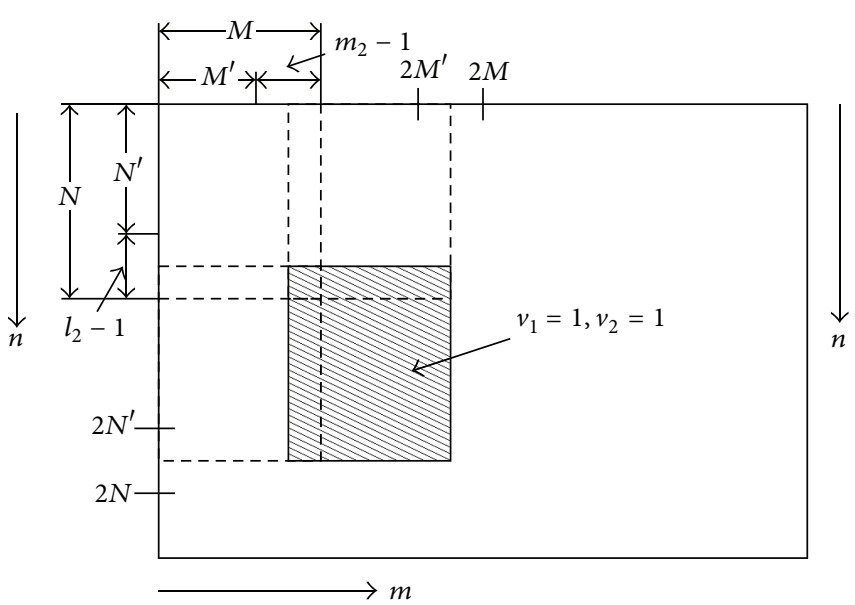

(d)

FIGURE 1: Four divided input submatrices are overlapped using the 2D overlap-save method: (a) the first submatrix, with parameters $v_{1}=$ $0, v_{2}=0$, is located on the top-left corner; (b) the second one $\left(v_{1}=1, v_{2}=0\right)$ overlaps by the first submatrix, and the size of the overlapping between them is $N$ times $m_{2}-1$; (c) the third divided input submatrix has parameters of $v_{1}=0, v_{2}=1$. It is overlapped by the first submatrix, and the size of the overlapping between them is $l_{2}-1$ times $M$; (d) the fourth one with $v_{1}=1, v_{2}=1$ is overlapped by the first submatrix, and the size of the overlapping between them is $l_{2}-1$ times $m_{2}-1$.

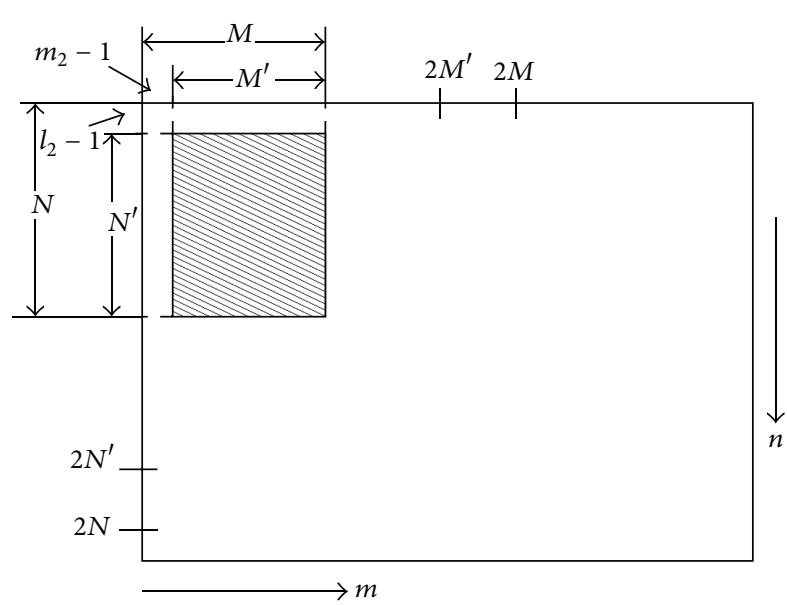

(a)

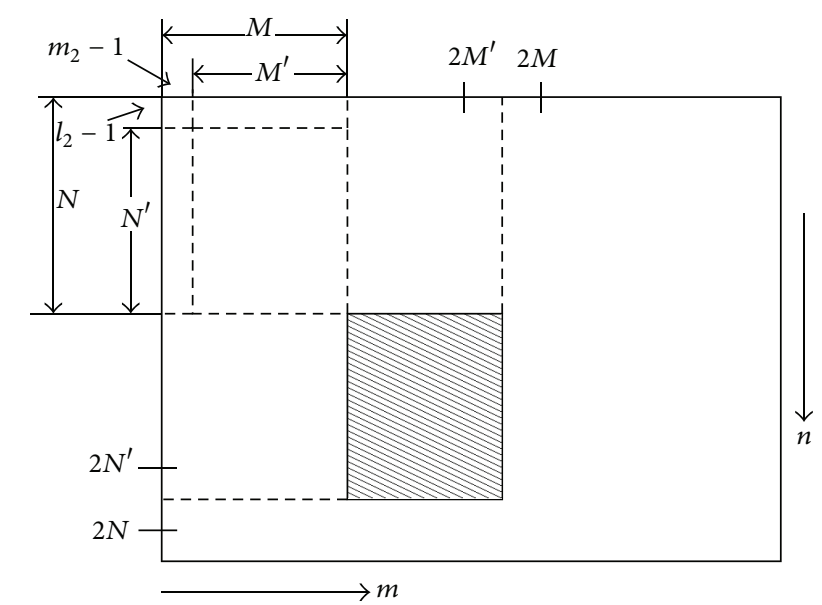

(b)

FIGURE 2: Two consecutive output submatrices: (a) the first block of $v_{1}=0$ and $v_{2}=0$ and (b) the second block of $v_{1}=1$ and $v_{2}=1$. 
Obviously, if the first $l_{2}-1$ data in rows and the first $m_{2}-1$ data in columns are discarded from the dashed rectangle, the darkened rectangle can be obtained. In the following, we will explain how to obtain the result of (4).

The output of the whole equation (4) can be represented in the following matrix:

$$
Y=\left(\begin{array}{cccc}
y_{0,0} & \cdots & \cdots & y_{0, m_{1}-1} \\
\vdots & \vdots & \vdots & \vdots \\
y_{l_{1}-1,0} & \cdots & \cdots & y_{l_{1}-1, m_{1}-1}
\end{array}\right)
$$

which is an $l_{1} \times m_{1}$ matrix.

So, (32) gives a $N^{\prime} \times M^{\prime}$ submatrix of matrix $Y$ (note that $N^{\prime}=N-l_{2}+1$ and that $\left.M^{\prime}=M-m_{2}+1\right)$ :

$$
\left(\begin{array}{cccc}
y_{l_{2}-1+v_{1} N^{\prime}, m_{2}-1+v_{2} M^{\prime}} & \cdots & \cdots & y_{l_{2}-1+v_{1} N^{\prime}, M-1+v_{2} M^{\prime}} \\
\vdots & \vdots & \vdots & \vdots \\
y_{N-1+v_{1} N^{\prime}, m_{2}-1+v_{2} M^{\prime}} & \cdots & \cdots & y_{N-1+v_{1} N^{\prime}, M-1+v_{2} M^{\prime}}
\end{array}\right) .
$$

Next, to obtain the whole result, we need to compute the circular convolution of two 2D sequences $x_{n_{1}+\left(v_{1}+1\right) N^{\prime}, n_{2}+\left(v_{2}+1\right) M^{\prime}}$ and $h_{n_{1}, n_{2}}^{\prime}\left(n_{1}=0,1, \ldots, N-1, n_{2}=0,1, \ldots, M-1\right)$. With the same reasoning as above, a consecutive submatrix of the matrix (34) is evinced as follows:

$$
\left(\begin{array}{cccc}
y_{N+v_{1} N^{\prime}, M+v_{2} M^{\prime}} & \cdots & \cdots & y_{N+v_{1} N^{\prime}, M+M^{\prime}-1+v_{2} M^{\prime}} \\
\vdots & \vdots & \vdots & \vdots \\
y_{N+N^{\prime}-1+v_{1} N^{\prime}, M+v_{2} M^{\prime}} & \cdots & \cdots & y_{N+N^{\prime}-1+v_{1} N^{\prime}, M+M^{\prime}-1+v_{2} M^{\prime}}
\end{array}\right) .
$$

The corresponding graphic description is indicated in Figure 2(b). We will darken the rectangle in Figure 2(b) to express the submatrix (35) and the dashed one on its upperleft-corner to be the graphic description of the submatrix (34).

Clearly, if $\left(v_{1}, v_{2}\right) \neq\left(v_{1}^{\prime}, v_{2}^{\prime}\right)$, then $\left(t_{1}+v_{1} N^{\prime}, t_{2}+\right.$ $\left.v_{2} M^{\prime}\right) \neq\left(t_{1}^{\prime}+v_{1}^{\prime} N^{\prime}, t_{2}^{\prime}+v_{2}^{\prime} M^{\prime}\right)$, where $l_{2}-1 \leq t_{1}, t_{1}^{\prime} \leq$ $N-1, m_{2}-1 \leq t_{2}$ and $t_{2}^{\prime} \leq M-1$. Let $[x]$ represent the largest integer that is less than or equal to the real number $x$; therefore, when $v_{1}=0,1, \ldots,\left[\left(l_{1}-N\right) / N^{\prime}\right]$ and $v_{2}=0,1, \ldots,\left[\left(m_{1}-M\right) / M^{\prime}\right]$, we will obtain all $N^{\prime} \times M^{\prime}$ submatrices of the matrix $Y$. If $l_{1}=s_{1} N^{\prime}+N$ and $m_{1}=s_{2} M^{\prime}+$ $M$, then $\left[\left(l_{1}-N\right) / N^{\prime}\right]=\left(l_{1}-N\right) / N^{\prime}$ and $\left[\left(m_{1}-M\right) / M^{\prime}\right]=$ $\left(m_{1}-M\right) / M^{\prime}$. The input matrix $x_{n_{1}, n_{2}}$ will be divided with no remainder. Figure 3 displays a series of small rectangles. Each rectangle stands for one $N^{\prime} \times M^{\prime}$ submatrix. The entire matrix $Y$ can be obtained by combining them.

In Figure 3, there is a white $\Gamma$-type area indicating that there are no output data. Two calculation methods can be applied to obtain the data. The first way is by using a direct method because only a few computations are needed. Alternatively, we can compute the data of $\Gamma$-type area with the overlap-save method proposed above, if $N^{\prime}=l_{2}-1$ and $M^{\prime}=$ $m_{2}-1$. The detail of it is presented in the Technical Report [18]. The entire result is completed and is demonstrated in Figure 4 graphically.

The two-dimensional overlap-save method is summarized as follows.

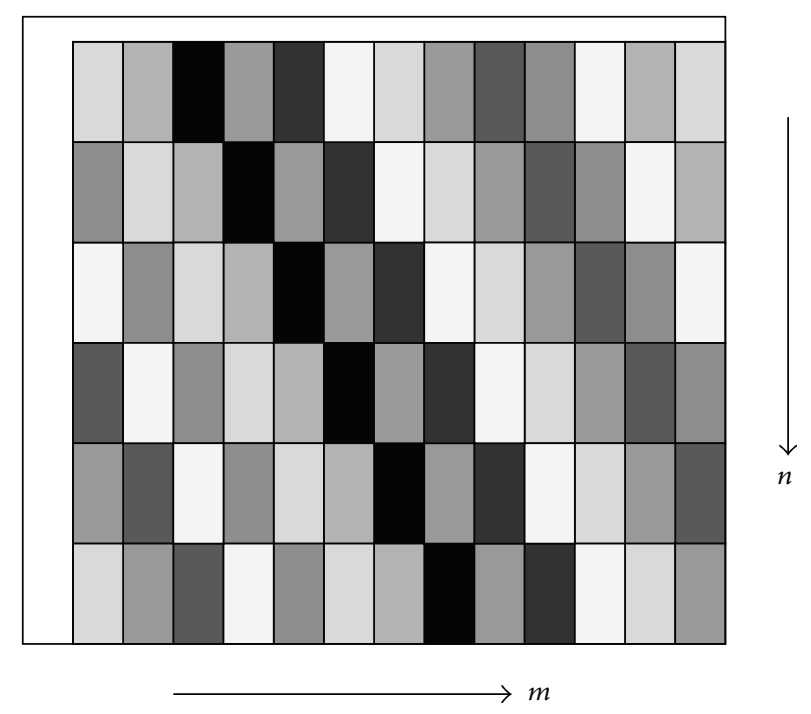

Figure 3: All $N^{\prime} \times M^{\prime}$ submatrices of the output, except the boundary of $\Gamma$-type area.

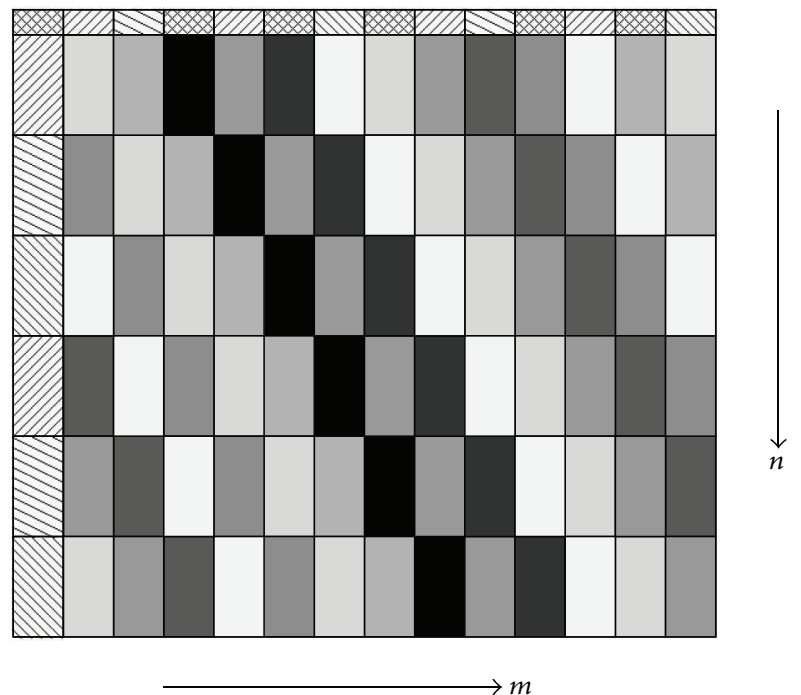

Figure 4: The full result of (4) computed by 2D overlap-save method, where $l_{1}=s_{1} N^{\prime}+N, m_{1}=s_{2} M^{\prime}+M$, and $N^{\prime}+l_{2}-1=$ $N<l_{1}$ and $M^{\prime}+m_{2}-1=M<m_{1}$.

(i) Select two positive integers $N^{\prime}$ and $M^{\prime}$, so that $N^{\prime}+$ $l_{2}-1=N<l_{1}$ and $M^{\prime}+m_{2}-1=M<m_{1}$. Either $N$ or $M$ is given by an integer power of 2 for using an FFT; that is, $N=2^{d_{1}}$ and $M=2^{d_{2}}$ where $d_{1}$ and $d_{2}$ are two positive integers.

(ii) Let $h_{n_{1}, n_{2}}^{\prime}$ satisfy (28).

(iii) Compute the circular convolution of two sequences $x_{n_{1}+v_{1} N^{\prime}, n_{2}+v_{2} M^{\prime}}$ and $h_{n_{1}, n_{2}}^{\prime}\left(n_{1}=0,1, \ldots, N-1, n_{2}=\right.$ $0,1, \ldots, M-1)$. When $t_{1}=l_{2}-1, \ldots, N-1, t_{2}=$ $m_{2}-1, \ldots, M-1$ and $v_{1}=0,1, \ldots,\left[\left(l_{1}-N\right) / N^{\prime}\right]$, $v_{2}=0,1, \ldots,\left[\left(m_{1}-M\right) / M^{\prime}\right]$, we obtain all $N^{\prime} \times M^{\prime}$ submatrices of the matrix of $Y$ whose form is as (34). 
If $l_{1}=s_{1} N^{\prime}+N$ and $m_{1}=s_{2} M^{\prime}+M$, then $\left[\left(l_{1}-\right.\right.$ $\left.N) / N^{\prime}\right]=\left(l_{1}-N\right) / N^{\prime}$ and $\left[\left(m_{1}-M\right) / M^{\prime}\right]=\left(m_{1}-\right.$ $M) / M^{\prime}$. The input matrix $x_{n_{1}, n_{2}}$ will be divided with no remainder.

(iv) For $t_{1}=0,1, \ldots, l_{2}-2, t_{2}=0,1, \ldots, m_{1}-1$, and $t_{1}=$ $0,1, \ldots, l_{1}-1, t_{2}=0,1, \ldots, m_{2}-2$, compute $y_{t_{1}, t_{2}}$ by using the direct method.

If $N^{\prime}=l_{2}-1$ and $M^{\prime}=m_{2}-1$, we will add zeros to the sequence $x_{n_{1}, n_{2}}$ so that the $2 \mathrm{D}$ sequences are of lengths $l_{1}+N^{\prime}$ and $m_{1}+M^{\prime}$. Then, we will compute $y_{n_{1}, n_{2}}\left(t_{1}=0,1, \ldots, l_{2}-2, t_{2}=0,1, \ldots, m_{1}-1\right.$, and $\left.t_{1}=0,1, \ldots, l_{1}-1, t_{2}=0,1, \ldots, m_{2}-2\right)$ by using an overlap-save method. The detail of it is presented in the Technical Report [18].

We will introduce the way of using FNTT and 2D overlap-save method with two examples. One is numerical computation and the other is an experiment of extracting the contour of a Chinese handwritten character.

\section{Comparison of the Numbers of Multiplication}

Let $N=N^{\prime}+l_{2}-1<l_{1}, M=M^{\prime}+m_{2}-1<m_{1}, N^{\prime}=l_{2}-1$, $M^{\prime}=m_{2}-1, l_{2}=2^{u}+1, m_{2}=2^{u^{\prime}}+1$, and $l_{1}=s_{1} N^{\prime}+N$, $m_{1}=s_{2} M^{\prime}+M$. Suppose that $a_{1}$ and $a_{2}$ satisfy $2^{a_{1}-1}<l_{1}+$ $l_{2}-1 \leq 2^{a_{1}}=N_{1}$ and $a^{a_{2}-1}<m_{1}+m_{2}-1 \leq 2^{a_{2}}=N_{2}$. Let $L_{1}$, $L_{2}$, and $L_{3}$ denote the numbers of multiplication necessary for computing the convolution (4) by using direct method, FNTT, or FFT to calculate (4) (using Proposition 1) and $2 \mathrm{D}$ overlap-save method, respectively. In [18], we compared the number of multiplications necessary for computing the convolution (4) of two $2 \mathrm{D}$ sequences $x_{n_{1}, n_{2}}\left(n_{1}=0,1, \ldots, l_{1}-\right.$ $\left.1, n_{2}=0,1, \ldots, m_{1}-1\right)$ and $h_{n_{1}, n_{2}}\left(n_{1}=0,1, \ldots, l_{2}-1\right.$, $\left.n_{2}=0,1, \ldots, m_{2}-1\right)$ by three different methods.

We proved the following results:

(1) If $l_{2}<l_{1}, m_{2}<m_{1}$, then

$$
\begin{aligned}
L_{1}= & \frac{l_{2} m_{2}\left(l_{2}+1\right)\left(m_{2}+1\right)}{4}+\left(m_{1}-m_{2}\right) m_{2} \frac{l_{2}\left(l_{2}+1\right)}{2} \\
& +\left(l_{1}-l_{2}\right) l_{2} \frac{m_{2}\left(m_{2}+1\right)}{2}+\left(l_{1}-l_{2}\right)\left(m_{1}-m_{2}\right) l_{2} m_{2} .
\end{aligned}
$$

Also, if $l_{1}=m_{1}=l, l_{2}=m_{2}=l^{\prime}$, then

$$
L_{1}=\left(\frac{l^{\prime}\left(2 l-l^{\prime}+1\right)}{2}\right)^{2} .
$$

(2) Consider

$$
L_{2}=2^{a_{1}+a_{2}-1}\left(3 a_{1}+3 a_{2}+2\right) \text {. }
$$

(3) If $l_{1}=m_{1}=2^{u+h}, l_{2}=m_{2}=2^{u}+1, N=M=2^{u+1}$, and $N^{\prime}=M^{\prime}=2^{u}$, where $u \geq 3, h \geq 2$, then

$$
\begin{aligned}
& L_{1}=\left(\left(2^{u}+1\right) 2^{u-1}\left(2^{h+1}-1\right)\right)^{2}, \\
& L_{2}=2^{2 u+2 h+1}(6 u+6 h+8), \\
& L_{3}=2\left(2^{u+h}\right)^{2}(6 u+8) .
\end{aligned}
$$

In this section, we will suppose that $N^{\prime} \neq l_{2}-1, M^{\prime} \neq m_{2}-$ 1. Let $N=N^{\prime}+l_{2}-1<l_{1}, M=M^{\prime}+m_{2}-1<m_{1}$, and $l_{1}=s_{1} N^{\prime}+N, m_{1}=s_{2} M^{\prime}+M$. For $t_{1}=0,1, \ldots, l_{2}-2, t_{2}=$ $0,1, \ldots, m_{1}-1$ and $t_{1}=0,1, \ldots, l_{1}-1, t_{2}=0,1, \ldots, m_{2}-2$, we computer $y_{t_{1}, t_{2}}$ by using the direct method.

We have the following proposition.

Proposition 6. If $l_{2}<l_{1}, m_{2}<m_{1}$, then

$$
\begin{aligned}
L=\frac{l_{2} m_{2}}{4} & \left(\left(l_{2}-1\right)\left(m_{2}-1\right)+2\left(l_{2}-1\right)\right. \\
& \left.\times\left(m_{1}-m_{2}+1\right)+2\left(m_{2}-1\right)\left(l_{1}-l_{2}+1\right)\right),
\end{aligned}
$$

where $L$ denotes the number of multiplication necessary for computing $y_{n_{1}, n_{2}}$ by using the direct method, $t_{1}=0,1, \ldots, l_{2}-2$, $t_{2}=0,1, \ldots, m_{1}-1$, and $t_{1}=0,1, \ldots, l_{1}-1, t_{2}=0,1, \ldots, m_{2}-$ 2.

$$
\begin{array}{r}
\text { Also, if } l_{1}=m_{1}=l \text { and } l_{2}=m_{2}=l^{\prime} \text {, then } \\
L=\frac{l^{\prime 2}\left(l^{\prime}-1\right)}{4}\left(4 l-3 l^{\prime}+3\right) .
\end{array}
$$

Proof. Let $I, I I$, and $I I I$ denote the number of multiplication necessary for computing the $A, B$, and $C$ by direct method, respectively, where

$$
A=\left(\begin{array}{ccc}
y_{0,0} & \cdots & y_{0, m_{2}-2} \\
\vdots & \vdots & \vdots \\
y_{l_{2}-2,0} & \cdots & y_{l_{2}-2, m_{2}-2}
\end{array}\right)
$$

$$
B=\left(\begin{array}{ccc}
y_{0, m_{2}-1} & \cdots & y_{0, m_{1}-1} \\
\vdots & \vdots & \vdots \\
y_{l_{2}-2, m_{2}-1} & \cdots & y_{l_{2}-2, m_{1}-1}
\end{array}\right)
$$

$$
C=\left(\begin{array}{ccc}
y_{l_{2}-1,0} & \cdots & y_{l_{2}-1, m_{2}-2} \\
\vdots & \vdots & \vdots \\
y_{l_{2}-1,0} & \cdots & y_{l_{2}-1, m_{2}-2}
\end{array}\right)
$$


Then

$$
\begin{aligned}
I & =\left(1+2+\cdots+\left(m_{2}-1\right)\right)\left(1+2+\cdots+\left(l_{2}-1\right)\right) \\
& =\frac{l_{2} m_{2}\left(l_{2}-1\right)\left(m_{2}-1\right)}{4}, \\
I I & =m_{2}\left(1+2+\cdots+\left(l_{2}-1\right)\right)\left(m_{1}-1-\left(m_{2}-2\right)\right) \\
& =\frac{l_{2} m_{2}\left(l_{2}-1\right)\left(m_{1}-m_{2}+1\right)}{2}, \\
I I I & =l_{2}\left(1+2+\cdots+\left(m_{2}-1\right)\right)\left(l_{1}-1-\left(l_{2}-2\right)\right) \\
& =\frac{l_{2} m_{2}\left(m_{2}-1\right)\left(l_{1}-l_{2}+1\right)}{2} .
\end{aligned}
$$

So,

$$
\begin{aligned}
L=I+I I & +I I I \\
=\frac{l_{2} m_{2}}{4} & \left(\left(l_{2}-1\right)\left(m_{2}-1\right)+2\left(l_{2}-1\right)\left(m_{1}-m_{2}+1\right)\right. \\
& \left.+2\left(m_{2}-1\right)\left(l_{1}-l_{2}+1\right)\right) .
\end{aligned}
$$

Clearly, if $l_{1}=m_{1}=l$ and $l_{2}=m_{2}=l^{\prime}$, then (41) holds. This completes the proof.

Proposition 7. If $l_{1}=m_{1}=2^{u+h}, l_{2}=m_{2}=2^{u}+1, N=$ $M=2^{u+2}$, and $N^{\prime}=M^{\prime}=3 \cdot 2^{u}$, where $u \geq 3, h \equiv 0(\bmod 2)$, $h \geq 4$, then

$$
\begin{aligned}
L_{4}= & \left(2^{u}+1\right)^{2} 2^{2(u-1)}\left(2^{h+2}-3\right) \\
& +\left(\frac{4\left(2^{h-2}-1\right)}{3}+1\right)^{2} 2^{2(u+2)}(3 u+7),
\end{aligned}
$$

where $L_{4}$ denotes the number of multiplication necessary for computing (4) by using 2D overlap-save method.

Proof. Because $N^{\prime} \neq l_{2}-1, M^{\prime} \neq m_{2}-1, l_{1}=m_{1}=2^{u+h}$, and $l_{2}=m_{2}=2^{u}+1$, by $(41)$, we have

$$
\begin{aligned}
L & =\left(2^{u}+1\right)^{2} \cdot 2^{u-2}\left(2^{u+h+2}-3\left(2^{u}+1\right)+3\right) \\
& =\left(2^{u}+1\right)^{2} 2^{2(u-1)}\left(2^{h+2}-3\right) .
\end{aligned}
$$

Compute the cyclic convolution of two sequences $x_{n_{1}+v_{1} N^{\prime}, n_{2}+v_{2} M^{\prime}}$ and $h_{n_{1}, n_{2}}^{\prime}\left(n_{1}=0,1, \ldots, 2^{u+2}-1\right.$, $\left.n_{2}=0,1, \ldots, 2^{u+2}-1\right)$; that is, compute matrix (35), by using FNNT. It is well known that using FNTT to calculate the (35) requires

$$
N M\left(\frac{3}{2} \log N M+1\right)=2^{2(u+2)}(3 u+7),
$$

where $N=M=2^{u+2}$.
When $N=M=2^{u+2}, N^{\prime}=M^{\prime}=3 \cdot 2^{u}, u \geq 3, h \equiv$ $0(\bmod 2)$, and $h \geq 4$, then

$$
\begin{aligned}
{\left[\frac{l_{1}-N}{N^{\prime}}\right] } & =\left[\frac{m_{1}-M}{M^{\prime}}\right]=\left[\frac{2^{u+h}-2^{u+2}}{3 \cdot 2^{u}}\right] \\
& =\left[\frac{4\left(2^{h-2}-1\right)}{3}\right]=\frac{4\left(2^{h-2}-1\right)}{3},
\end{aligned}
$$

where $4\left(2^{h-2}-1\right) / 3$ is a positive integer.

So, the input matrix $x_{n_{1}, n_{2}}$ will be divided with no remainder. When $v_{1}=0,1, \ldots,\left[\left(l_{1}-N\right) / N^{\prime}\right]$ and $v_{2}=$ $0,1, \ldots,\left[\left(m_{1}-M\right) / M^{\prime}\right]$, we obtain all matrices as (35). Thus,

$$
\begin{aligned}
L_{4}= & \left(2^{u}+1\right)^{2} 2^{2(u-1)}\left(2^{h+2}-3\right) \\
& +\left(\frac{4\left(2^{h-2}-1\right)}{3}+1\right)^{2} 2^{2(u+2)}(3 u+7),
\end{aligned}
$$

equal to (45) holds. This completes the proof.

Example 8. Suppose that $u=h=4$; that is, $l_{1}=m_{1}=256$, $l_{2}=m_{2}=17, N=M=64$, and $N^{\prime}=M^{\prime}=48$; then,

$$
L_{4}=3073856 \text {. }
$$

If $l_{1}=m_{1}=256, l_{2}=m_{2}=17, N=M=32$, and $N^{\prime}=M^{\prime}=$ 16 , then

$$
L_{3}=4194304 .
$$

This example shows that $L_{4}<L_{3}$ in some particular cases.

\section{Experiments}

The theoretic description of the 2D overlap-save method has been provided in the preceding section. This section further examines how such a technique will be performed and how it will be applied to the wavelet transform. Particularly, we will use image processing as an example. The experiment will focus on the application of the 2D overlap-save method to the dyadic wavelet transform extracting the contours of Chinese handwriting.

5.1. Numerical Computation. Let $h_{n_{1}, n_{2}}$ denote a digital filter and let a two-dimensional sequence

$$
\left(h_{n_{1}, n_{2}}\right)=\left(\begin{array}{lll}
1 & 0 & 1 \\
1 & 1 & 0 \\
0 & 1 & 1
\end{array}\right)
$$

be the coefficients of the filter. A two-dimensional sequence

$$
\left(x_{n_{1}, n_{2}}\right)=\left(\begin{array}{cccccccc}
1 & 3 & 2 & -3 & 0 & 2 & 1 & 1 \\
3 & 1 & 0 & -1 & 2 & -2 & -1 & 0 \\
0 & -1 & 1 & 1 & 3 & 2 & 0 & -1 \\
2 & 3 & -1 & 1 & 0 & 1 & 1 & 0 \\
3 & -2 & 1 & 0 & -1 & 1 & 1 & 1 \\
-1 & 0 & 2 & 3 & 1 & 1 & 0 & 1 \\
1 & -1 & 0 & 1 & 0 & 1 & -1 & 0 \\
-1 & 0 & 1 & 1 & 0 & 1 & 0 & 1
\end{array}\right)
$$


represents the input of the filter. In this example, because $l_{1}=$ $m_{1}=8$ and $l_{2}=m_{2}=3$, the length of input matrix is $8 \times 8$ and that of the filter is $3 \times 3$.

Using the overlap-save method to calculate the output of the filter

$$
\begin{gathered}
y_{n_{1}, n_{2}}=\sum_{\substack{k_{1}=0 \\
0 \leq n_{1}-k_{1}<8 \\
0 \leq n_{2}-k_{2}<8}}^{2} \sum_{k_{1}=0}^{2} h_{k_{1}, k_{2}} x_{n_{1}-k_{1}, n_{2}-k_{2}} \\
n_{1}=0,1,2,3,4,5,6,7, \quad n_{2}=0,1,2,3,4,5,6,7,
\end{gathered}
$$

means that the elements of the matrix

$$
Y=\left(\begin{array}{cccc}
y_{0,0} & y_{0,1} & \cdots & y_{0,7} \\
y_{1,0} & y_{1,1} & \cdots & y_{1,7} \\
\vdots & \vdots & \vdots & \vdots \\
y_{7,0} & y_{7,1} & \cdots & y_{7,7}
\end{array}\right)
$$

need to be computed.

The solution is given below.

Since $l_{1}=m_{1}=8, l_{2}=m_{2}=3$, by selecting $N^{\prime}=l_{2}-1=$ 2 , and $M^{\prime}=m_{2}-1=2$, we have $N=M=4$. In order to use the FNTT, the input matrix is divided into 9 submatrices of size $4 \times 4$. Each consecutive submatrix is partly overlapped with the size of $2 \times 2$ pixels. In addition, zeros of size $1 \times 1$ are added to the filtering sequence to ensure that its length is equivalent to that of the submatrix. Therefore, the filtering sequence $h_{n_{1}, n_{2}}$ is extended to $h_{n_{1}, n_{2}}^{\prime}$; that is,

$$
h_{n_{1}, n_{2}}^{\prime}= \begin{cases}h_{n_{1}, n_{2}} & \text { if } n_{1}=0,1,2, n_{2}=0,1,2, \\ 0 & \text { if } n_{1}=3 \text { or } n_{2}=3,\end{cases}
$$

where $n_{1}=0,1,2,3, n_{2}=0,1,2,3$.

Next, we compute the circular convolution of the two sequences $x_{n_{1}+2 v_{1}, n_{2}+2 v_{2}}$ and $h_{n_{1}, n_{2}}^{\prime}\left(n_{1}=0,1,2,3, n_{2}=\right.$ $0,1,2,3)$,

$$
\begin{aligned}
\bar{y}_{t_{1}, t_{2}} & =\sum_{n_{1}=0}^{3} \sum_{n_{2}=0}^{3} h_{n_{1}, n_{2}}^{\prime} x_{\left\langle t_{1}-n_{1}\right\rangle_{4}+2 v_{1},\left\langle t_{2}-n_{2}\right\rangle_{4}+2 v_{2}} \\
& =\sum_{n_{1}=0}^{2} \sum_{n_{2}=0}^{2} h_{n_{1}, n_{2}} x_{\left\langle t_{1}-n_{1}\right\rangle_{4}+2 v_{1},\left\langle t_{2}-n_{2}\right\rangle_{4}+2 v_{2}},
\end{aligned}
$$

where $t_{1}=0,1,2,3, t_{2}=0,1,2,3$.

When $t_{1}=2,3, t_{2}=2,3$, we arrive at the following result:

$$
\begin{aligned}
\bar{y}_{t_{1}, t_{2}} & =\sum_{n_{1}=0}^{2} \sum_{n_{2}=0}^{2} h_{n_{1}, n_{2}} x_{t_{1}-n_{1}+2 v_{1}, t_{2}-n_{2}+2 v_{2}} \\
& =y_{t_{1}+2 v_{1}, t_{2}+2 v_{2}} .
\end{aligned}
$$

After choosing all the parameters of $v_{1}=0,1,2, v_{2}=$ $0,1,2\left(\left[\left(l_{1}-N\right) / N^{\prime}\right]=\left[\left(m_{1}-M\right) / M^{\prime}\right]=[(8-4) / 2]=2\right)$, all $2 \times 2$ submatrices of the matrix $Y$, defined in (34), will be obtained as follows:

$$
\begin{aligned}
& \left(\begin{array}{ll}
y_{2,2} & y_{2,3} \\
y_{3,2} & y_{3,3}
\end{array}\right)\left(\begin{array}{ll}
y_{2,4} & y_{2,5} \\
y_{3,4} & y_{3,5}
\end{array}\right)\left(\begin{array}{ll}
y_{2,6} & y_{2,7} \\
y_{3,6} & y_{3,7}
\end{array}\right) \\
& \left(\begin{array}{ll}
y_{4,2} & y_{4,3} \\
y_{5,2} & y_{5,3}
\end{array}\right)\left(\begin{array}{ll}
y_{4,4} & y_{4,5} \\
y_{5,4} & y_{5,5}
\end{array}\right)\left(\begin{array}{ll}
y_{4,6} & y_{4,7} \\
y_{5,6} & y_{5,7}
\end{array}\right) \\
& \left(\begin{array}{ll}
y_{6,2} & y_{6,3} \\
y_{7,2} & y_{7,3}
\end{array}\right)\left(\begin{array}{ll}
y_{6,4} & y_{6,5} \\
y_{7,4} & y_{7,5}
\end{array}\right)\left(\begin{array}{ll}
y_{6,6} & y_{6,7} \\
y_{7,6} & y_{7,7}
\end{array}\right) .
\end{aligned}
$$

For $y_{t_{1}, t_{2}}\left(t_{1}=0,1, t_{2}=0,1,2,3,4,5,6,7, t_{1}=\right.$ $\left.0,1,2,3,4,5,6,7, t_{2}=0,1\right)$, we can compute it by using an overlap-save method. The detail of it is presented in the Technical Report [18].

The fast number theoretic transform (FNTT) is now applied to the implementation of the circular convolution

$$
\begin{array}{r}
\bar{y}_{t_{1}, t_{2}}=\sum_{n_{1}=0}^{3} \sum_{n_{2}=0}^{3} h_{n_{1}, n_{2}} x_{\left\langle t_{1}-n_{1}\right\rangle_{4},\left\langle t_{2}-n_{2}\right\rangle_{4}}, \\
t_{1}=0,1,2,3, \quad t_{2}=0,1,2,3 .
\end{array}
$$

The computation is performed in the following five steps shown in Figure 5.

Step 1 (choosing $M)$. Since

$$
\left|\bar{y}_{t_{1}, t_{2}}\right| \leq\left|x_{n_{1}, n_{2}}\right|{ }_{\max } \sum_{k_{1}=0}^{3} \sum_{k_{2}=0}^{3}\left|h_{k_{1}, k_{2}}\right|=3 \times 6=18,
$$

we can select $m=257=2^{8}+1$ so that $18<257 / 2$, where 257 is a prime. Clearly, $16^{4} \equiv 1(\bmod 257)$, and $16^{j} \not \equiv 1(\bmod$ $257)$, for $1 \leq j \leq 3$.

Thus,

$$
\begin{array}{r}
X_{k_{1}, k_{2}} \equiv \sum_{n_{1}=0}^{3} \sum_{n_{2}=0}^{3} x_{n_{1}, n_{2}} 16^{n_{1} k_{1}} \cdot 16^{n_{2} k_{2}}(\bmod 257), \\
k_{1}=0,1,2,3, \quad k_{2}=0,1,2,3
\end{array}
$$

is a $2 \mathrm{D}$ number theoretic transform. It has the following inverse transform:

$$
\begin{array}{r}
x_{n_{1}, n_{2}} \equiv 4^{-1} \cdot 4^{-1} \sum_{k_{1}=0}^{3} \sum_{k_{2}=0}^{3} X_{k_{1}, k_{2}}(-16)^{n_{1} k_{1}}(-16)^{n_{2} k_{2}}(\bmod 257), \\
n_{1}=0,1,2,3, \quad n_{2}=0,1,2,3,
\end{array}
$$

where $4^{-1}$ denotes an integer so that $\left(4^{-1}\right) \cdot 4 \equiv 1(\bmod 257)$. 


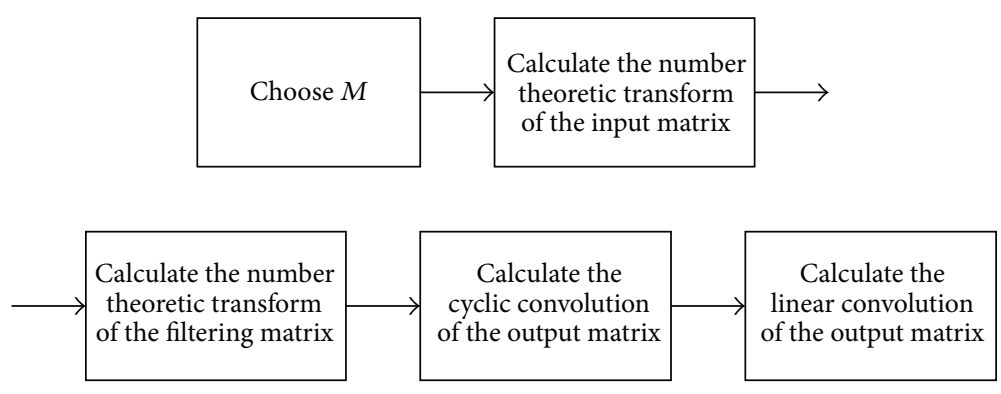

FIGURE 5: Five steps of applying FNTT to calculate the output of (54).

Step 2 (calculating the number theoretic transform of input matrix by the FNTT). For computational convenience, here, one writes

$$
\begin{array}{r}
U_{0, k_{2}} \equiv \sum_{n_{2}=0}^{3} x_{0, n_{2}} 16^{n_{2} k_{2}}, \quad U_{1, k_{2}} \equiv \sum_{n_{2}=0}^{3} x_{1, n_{2}} 16^{n_{2} k_{2}}(\bmod 257) \\
U_{2, k_{2}} \equiv \sum_{n_{2}=0}^{3} x_{2, n_{2}} 16^{n_{2} k_{2}}, \quad U_{3, k_{2}} \equiv \sum_{n_{2}=0}^{3} x_{3, n_{2}} 16^{n_{2} k_{2}}(\bmod 257) \\
k_{2}=0,1,2,3 .
\end{array}
$$

Then, (62) becomes

$$
\begin{array}{r}
X_{k_{1,0}} \equiv \sum_{n_{1}=0}^{3} U_{n_{1,0}} 16^{n_{1} k_{1}}, \quad X_{k_{1,1}} \equiv \sum_{n_{1}=0}^{3} U_{n_{1,1}} 16^{n_{1} k_{1}}(\bmod 257) \\
X_{k_{1,2}} \equiv \sum_{n_{1}=0}^{3} U_{n_{1,2}} 16^{n_{1} k_{1}}, \quad X_{k_{1,3}} \equiv \sum_{n_{1}=0}^{3} U_{n_{1,3}} 16^{n_{1} k_{1}}(\bmod 257) \\
k_{1}=0,1,2,3 .
\end{array}
$$

Use the FNTT to calculate (64) and (65).

So,

$$
\begin{aligned}
& \left(\begin{array}{llll}
U_{0,0} & U_{0,1} & U_{0,2} & U_{0,3} \\
U_{1,0} & U_{1,1} & U_{1,2} & U_{1,3} \\
U_{2,0} & U_{2,1} & U_{2,2} & U_{2,3} \\
U_{3,0} & U_{3,1} & U_{3,2} & U_{3,3}
\end{array}\right) \\
& \equiv\left(\begin{array}{cccc}
3 & 95 & 3 & -97 \\
3 & 35 & 3 & -29 \\
1 & -33 & 1 & 31 \\
5 & 35 & -3 & -29
\end{array}\right)(\bmod 257), \\
X & \left(\begin{array}{cccc}
X_{0,0} & X_{0,1} & X_{0,2} & X_{0,3} \\
X_{1,0} & X_{1,1} & X_{1,2} & X_{1,3} \\
X_{2,0} & X_{2,1} & X_{2,2} & X_{2,3} \\
X_{3,0} & X_{3,1} & X_{3,2} & X_{3,3}
\end{array}\right) \\
& \equiv\left(\begin{array}{cccc}
12 & 132 & 4 & -124 \\
-30 & 128 & 98 & -128 \\
-4 & -8 & 4 & -8 \\
34 & 128 & -94 & -128
\end{array}\right)(\bmod 257) .
\end{aligned}
$$

Step 3 (calculating the number theoretic transform of the filter by the FNTT). The FNTT of the filter $h_{n_{1}, n_{2}}$ is written as

$$
\begin{array}{r}
H_{k_{1}, k_{2}} \equiv \sum_{n_{1}=0}^{3} \sum_{n_{2}=0}^{3} h_{n_{1}, n_{2}}^{\prime} 16^{n_{1} k_{1}} \cdot 16^{n_{2} k_{2}}(\bmod 257), \\
k_{1}=0,1,2,3, \quad k_{2}=0,1,2,3 .
\end{array}
$$

Similarly, we have

$$
\begin{aligned}
H & =\left(\begin{array}{llll}
H_{0,0} & H_{0,1} & H_{0,2} & H_{0,3} \\
H_{1,0} & H_{1,1} & H_{1,2} & H_{1,3} \\
H_{2,0} & H_{2,1} & H_{2,2} & H_{2,3} \\
H_{3,0} & H_{3,1} & H_{3,2} & H_{3,3}
\end{array}\right) \\
& \equiv\left(\begin{array}{cccc}
6 & 32 & 2 & -32 \\
32 & 0 & 2 & 34 \\
2 & -2 & 2 & -2 \\
-32 & -30 & 2 & 0
\end{array}\right)(\bmod 257)
\end{aligned}
$$

Step 4 (compute the number theoretic transform of the output of the filter). From the preceding three steps, we can compute the circular convolution of the filter by defining the product of $X$ and $H$ as

$$
\begin{aligned}
\bar{Y} & =\left(\begin{array}{llll}
Y_{0,0} & Y_{0,1} & Y_{0,2} & Y_{0,3} \\
Y_{1,0} & Y_{1,1} & Y_{1,2} & Y_{1,3} \\
Y_{2,0} & Y_{2,1} & Y_{2,2} & Y_{2,3} \\
Y_{3,0} & Y_{3,1} & Y_{3,2} & Y_{3,3}
\end{array}\right) \\
& \equiv X \odot H=\left(\begin{array}{cccc}
X_{0,0} H_{0,0} & X_{0,1} H_{0,1} & X_{0,2} H_{0,2} & X_{0,3} H_{0,3} \\
X_{1,0} H_{1,0} & X_{1,1} H_{1,1} & X_{1,2} H_{1,2} & X_{1,3} H_{1,3} \\
X_{2,0} H_{2,0} & X_{2,1} H_{2,1} & X_{2,2} H_{2,2} & X_{2,3} H_{2,3} \\
X_{3,0} H_{3,0} & X_{3,1} H_{3,1} & X_{3,2} H_{3,2} & X_{3,3} H_{3,3}
\end{array}\right) \\
& \equiv\left(\begin{array}{cccc}
72 & 112 & 8 & 113 \\
68 & 0 & -61 & 17 \\
-8 & 16 & 8 & 16 \\
-60 & 15 & 69 & 0
\end{array}\right)(\bmod 257),
\end{aligned}
$$

where

$$
\begin{array}{r}
Y_{k_{1}, k_{2}} \equiv \sum_{n_{1}=0}^{3} \sum_{n_{2}=0}^{3} \bar{y}_{n_{1}, n_{2}} 16^{n_{1} k_{1}} \cdot 16^{n_{2} k_{2}}(\bmod 257) \\
k_{1}=0,1,2,3, \quad k_{2}=0,1,2,3
\end{array}
$$


TABLE 1: Filtering coefficients $\left\{\psi_{k, l}^{2^{j}, 1}\right\},(j=2), \psi(r)$, are a quadratic spline wavelet.

\begin{tabular}{lcccc}
\hline$l / k$ & $k=0$ & $k=1$ & $k=2$ & $k=3$ \\
\hline$l=0$ & -0.0838140026 & -0.0472041145 & -0.0129809398 & -0.0012592132 \\
$l=1$ & -0.1416904181 & -0.0758706033 & -0.0196341425 & -0.0012698699 \\
$l=2$ & -0.0651057437 & -0.0327749476 & -0.0063385521 & -0.0000750836 \\
$l=3$ & -0.0088690016 & -0.0030044997 & -0.0001088651 & \\
\hline
\end{tabular}

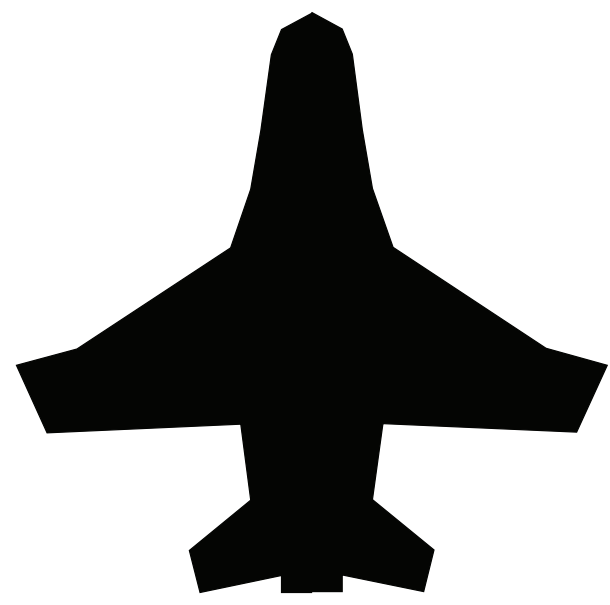

(a)

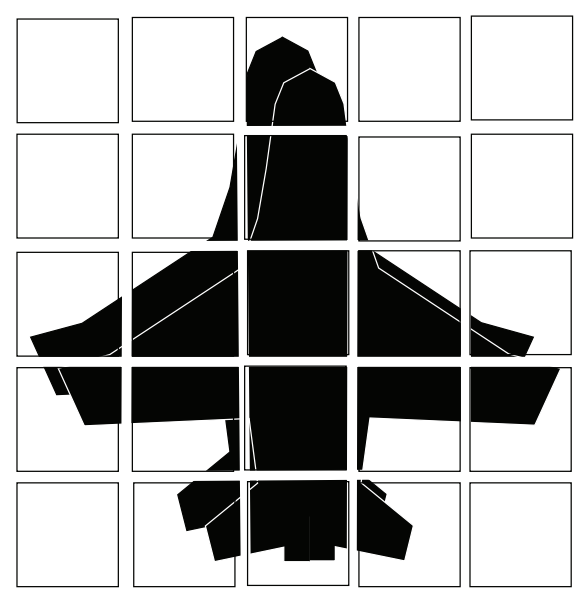

(b)

FIGURE 6: A 2-dimensional image of an aircraft was processed with 2D overlap-save method. (a) Original Chinese handwriting and (b) the divided up subimages of the character.

and the $\bar{y}_{n_{1}, n_{2}}\left(n_{1}=0,1,2,3, n_{2}=0,1,2,3\right)$ is the circular convolution of $(60)$.

Step 5 (calculate the output of the linear convolution). From (63), we deduce the inverse transform of (70):

$$
\begin{array}{r}
\bar{y}_{n_{1}, n_{2}} \equiv 4^{-1} \cdot 4^{-1} \sum_{k_{1}=0}^{3} \sum_{k_{2}=0}^{3} Y_{k_{1}, k_{2}}(-16)^{n_{1} k_{1}} \\
\cdot(-16)^{n_{2} k_{2}}(\bmod 257), \\
n_{1}=0,1,2,3, \quad n_{2}=0,1,2,3 .
\end{array}
$$

Using FNTT to calculate (71), we have

$$
\left(\begin{array}{llll}
\bar{y}_{0,0} & \bar{y}_{0,1} & \bar{y}_{0,2} & \bar{y}_{0,3} \\
\bar{y}_{1,0} & \bar{y}_{1,1} & \bar{y}_{1,2} & \bar{y}_{1,3} \\
\bar{y}_{2,0} & \bar{y}_{2,1} & \bar{y}_{2,2} & \bar{y}_{2,3} \\
\bar{y}_{3,0} & \bar{y}_{3,1} & \bar{y}_{3,2} & \bar{y}_{3,3}
\end{array}\right) \equiv\left(\begin{array}{cccc}
8 & 6 & 4 & 0 \\
1 & 7 & 13 & 1 \\
2 & 2 & 6 & 4 \\
1 & 5 & 5 & 7
\end{array}\right)(\bmod 257)
$$

Then, the linear convolution in (58) is obtained:

$$
\left(\begin{array}{ll}
y_{2,2} & y_{2,3} \\
y_{3,2} & y_{3,3}
\end{array}\right)=\left(\begin{array}{ll}
\bar{y}_{2,2} & \bar{y}_{2,3} \\
\bar{y}_{3,2} & \bar{y}_{3,3}
\end{array}\right)=\left(\begin{array}{ll}
6 & 4 \\
5 & 7
\end{array}\right) .
$$

5.2. Application to Image Processing. The proposed method can be applied to many fields such as image processing and pattern recognition. An example of the application to the image processing is illustrated in Figure 6. The original image shown in Figure 6(a) is a 2-dimensional image of an aircraft with a size of $256 \times 256$ pixels; that is, $l_{1}=m_{1}=$ 256. Our task is to extract its contours. The discrete wavelet transform with scale $s=2^{j}$ where $j=2$ was applied and the spline wavelet, described in (1), of size $17 \times 17$ was chosen; that is $l_{2}=m_{2}=17$. We use the filtering coefficients tabulated in Table 1. Using the 2D overlap-save method, this original image was divided into 25 sections, with each one having the dimension of $64 \times 64$ pixels; that is, we select $N=M=64, N^{\prime}=M^{\prime}=48,\left[\left(l_{1}-N\right) / N^{\prime}\right]=\left[\left(m_{1}-\right.\right.$ $\left.M) / M^{\prime}\right]=4$. (See Example 8 in Section 4.) Figure 6(b) shows these subimages. The overlapped part can be explicitly observed in each subimage.

To facilitate the presentation of the $2 \mathrm{D}$ overlap-save method, the procedure of it is diagonally displayed in Figure 7, where a series of the divided subimages are illustrated. The image in Figure 6(a) was equivalently divided into 25 sections by applying (27). In Figure 7(a), we represent 5 subimages of $v_{0}=0, v_{1}=0, v_{0}=1, v_{1}=1, v_{0}=2, v_{1}=2$ and $v_{0}=3, v_{1}=3$, and $v_{0}=4, v_{1}=4$, consecutively. The lower-right corner of each subimage is repeated in the upperleft corner of its consecutive subimage. Four subimages are displayed in Figure 7(b), and the parameters of $v_{0}=0, v_{1}=1$, $v_{0}=1, v_{1}=2, v_{0}=2, v_{1}=3$, and $v_{0}=3, v_{1}=4$ were chosen. In Figure 7(c), we selected 3 subimages of $v_{0}=0$, $v_{1}=2, v_{0}=1, v_{1}=3$, and $v_{0}=2, v_{1}=4$. There are 3 subimages of $v_{0}=0, v_{1}=3$ and $v_{0}=1, v_{1}=4 \mathrm{in}$ Figure 7(d). Figure 7(e) displays subimages of $v_{0}=0, v_{1}=4$. 


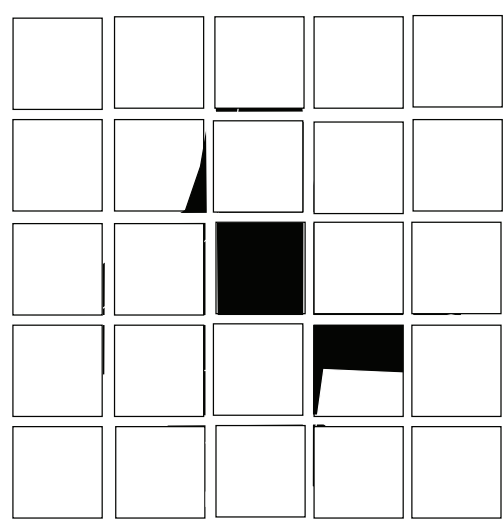

(a)

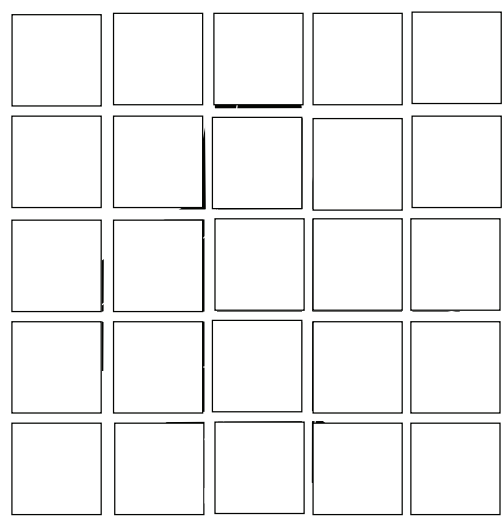

(d)

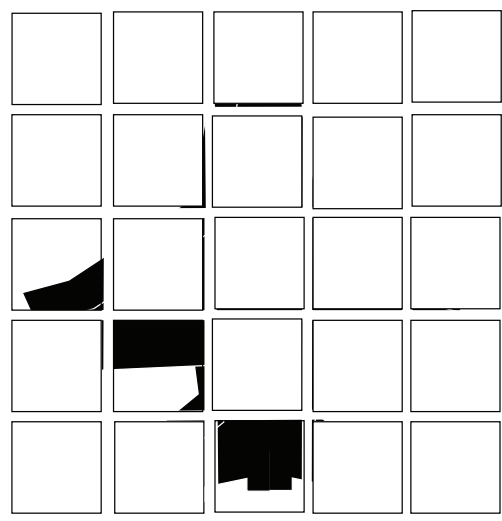

(g)

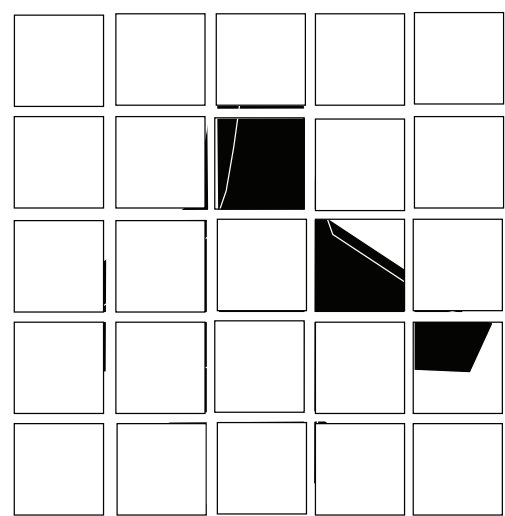

(b)

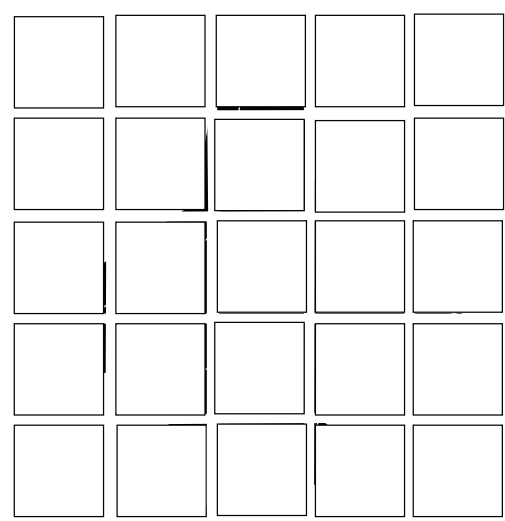

(e)

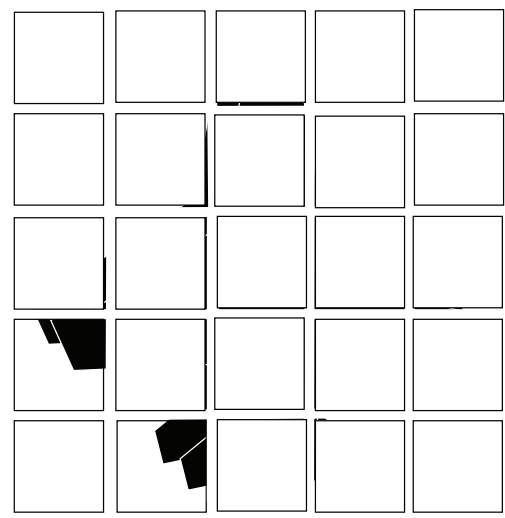

(h)

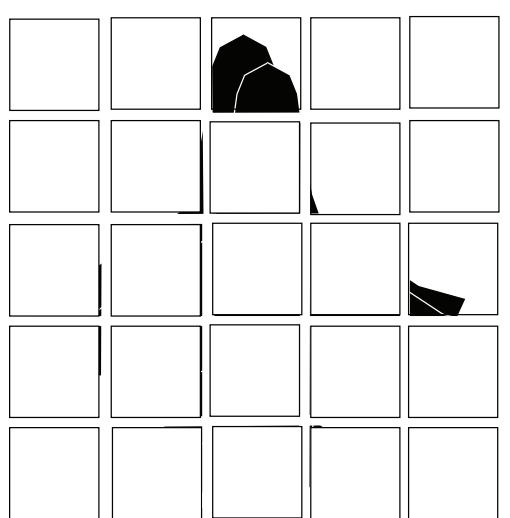

(c)

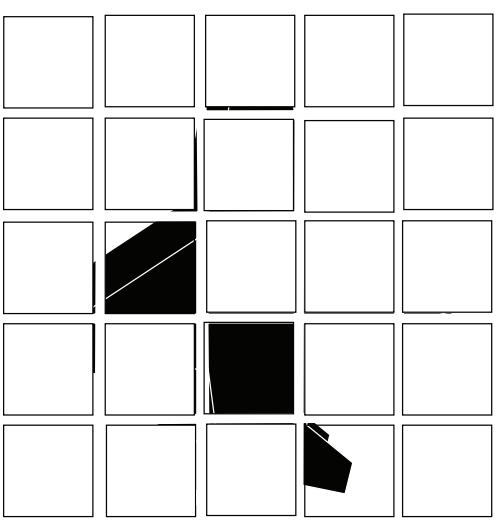

(f)

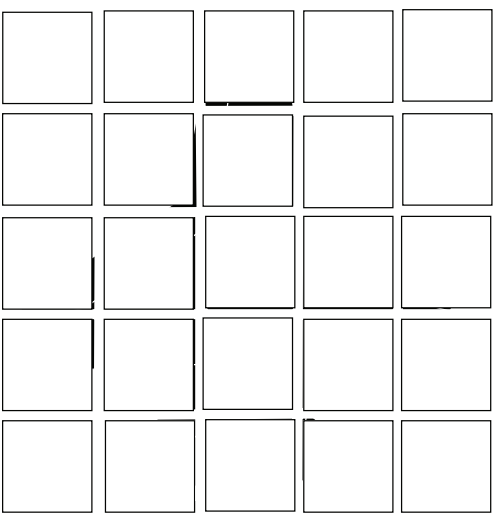

(i)

FIGURE 7: A 2-dimensional image of an aircraft is dividing into separated small subimages; the parameters of $v_{0}$ and $v_{1}$ are chosen as follows: (a) $v_{0}=0, v_{1}=0, v_{0}=1, v_{1}=1, v_{0}=2, v_{1}=2$ and $v_{0}=3, v_{1}=3$, and $v_{0}=4$ and $v_{1}=4$; (b) $v_{0}=0, v_{1}=1, v_{0}=1, v_{1}=2, v_{0}=2, v_{1}=3$, and $v_{0}=3$ and $v_{1}=4$; (c) $v_{0}=0, v_{1}=2, v_{0}=1, v_{1}=3$, and $v_{0}=2$ and $v_{1}=4$; (d) $v_{0}=0, v_{1}=3$, and $v_{0}=1$ and $v_{1}=4$; (e) $v_{0}=0$ and $v_{1}=4$; (f) $v_{0}=1, v_{1}=0, v_{0}=2, v_{1}=1, v_{0}=3, v_{1}=2$, and $v_{0}=4$ and $v_{1}=3 ;(\mathrm{g}) v_{0}=2, v_{1}=0, v_{0}=3, v_{1}=1$, and $v_{0}=4$ and $v_{1}=2 ;(\mathrm{h}) v_{0}=3, v_{1}=0$, and $v_{0}=4$ and $v_{1}=1$; and (i) $v_{0}=4$ and $v_{1}=0$.

In Figure 7(f), $v_{0}=1, v_{1}=0, v_{0}=2, v_{1}=1, v_{0}=3, v_{1}=2$, and $v_{0}=4, v_{1}=3$ were chosen. In Figure $7(\mathrm{~g})$ the parameters were decided upon $v_{0}=2, v_{1}=0, v_{0}=3, v_{1}=1$, and $v_{0}=4$, $v_{1}=2$. Two subimages are shown in Figure $7(\mathrm{~h})$ by selecting $v_{0}=3, v_{1}=0$, and $v_{0}=4, v_{1}=1$. There is one subimage of $v_{0}=4, v_{1}=0$ in Figure 7(i).
We select a 2D NTT

$$
X_{k_{1}, k_{2}} \equiv \sum_{n_{1}=0}^{63} \sum_{n_{2}=0}^{63} x_{n_{1}, n_{2}} 2^{n_{1} k_{1}} 2^{n_{2} k_{2}}\left(\bmod 2^{32}+1\right)
$$




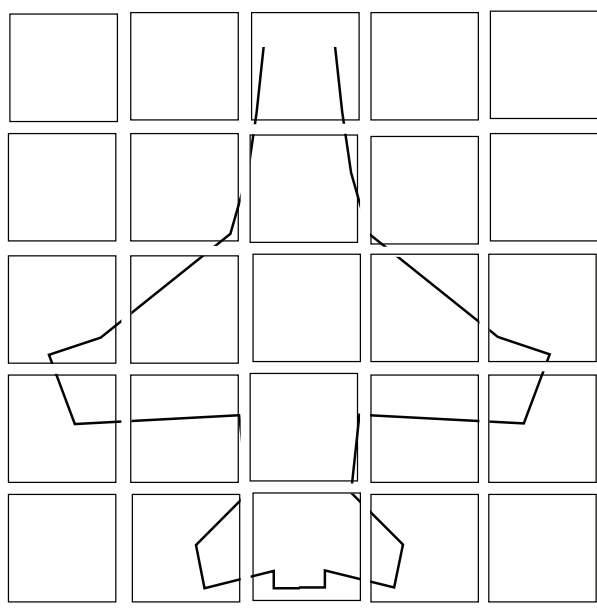

(a)

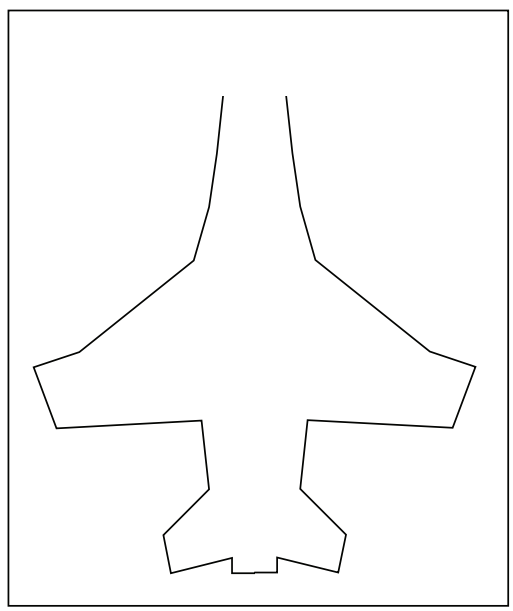

(c)
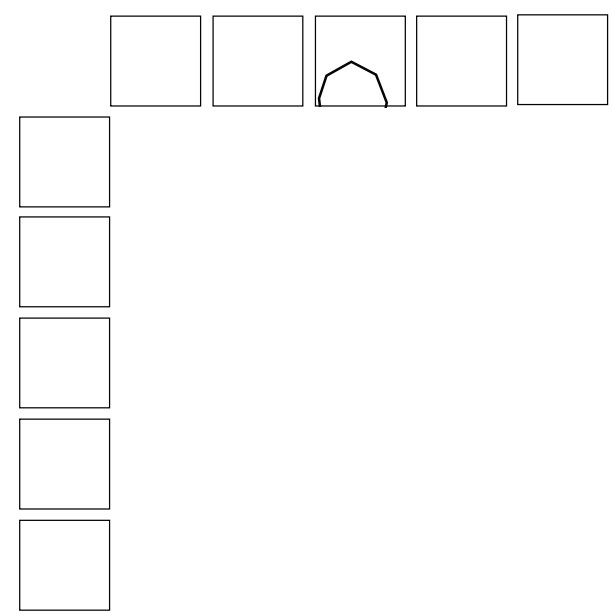

(b)

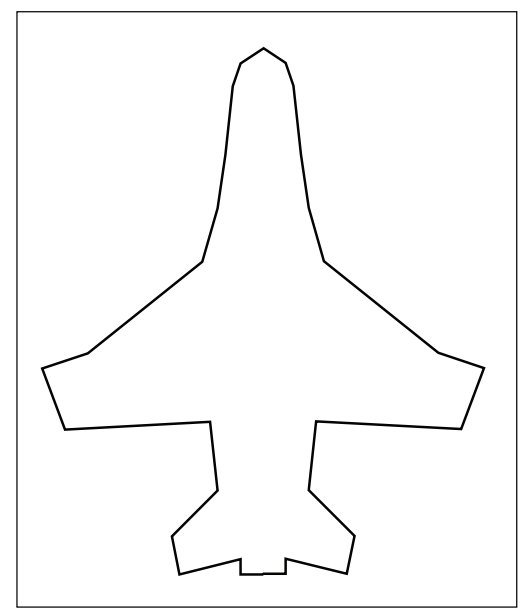

(d)

FIGURE 8: The linear outputs of 2D overlap-save method: (a) the outputs of the linear convolution of each subimages, (b) the left border and the upper border of the outputs of the image of aircraft, (c) the combined outputs of linear convolution, except $\Gamma$-type border, and (d) the entire contour extracted using 2D overlap-save method under wavelet transform.

which has inverse transform

$$
\begin{aligned}
x_{n_{1}, n_{2}} \equiv 64^{-1} 64^{-1} \sum_{k_{1}=0}^{63} \sum_{k_{2}=0}^{63} X_{k_{1}, k_{2}}\left(-2^{31}\right)^{n_{1} k_{1}} \\
\quad \times\left(-2^{31}\right)^{n_{2} k_{2}}\left(\bmod 2^{32}+1\right), \\
n_{1}=0,1, \ldots, 63, \quad n_{2}=0,1, \ldots, 63,
\end{aligned}
$$

where $64^{-1}$ denotes integer so that $\left(64^{-1}\right) 64 \equiv 1\left(\bmod 2^{32}+\right.$ $1)$, and $\left|x_{n_{1}, n_{2}}\right| \leq 255$.

The FNTT was applied to calculate the circular convolution of each subimage. After applying (31) to each of the subblocks, a series of segments of the contours were obtained.

By using (32), we can gradually reach the final result of the linear convolution. Figure 8(a) illustrates those pixels which satisfy (35), where $l_{2}=m_{2}=17, N=M=64, N^{\prime}=M^{\prime}=$ 48 , and $v_{1}, v_{2}=0,1,2,3,4$. Combining them together, the contours of the whole character were obtained and displayed in Figure 8(c) which excludes the $\Gamma$-type border. Next, the
$\Gamma$-type border was computed by direct method. Figure 8(b) shows the result. Combining this result with that shown in Figure 8(c), the entire contours of the Chinese handwriting were combined. Figure $8(\mathrm{~d})$ represents the entire contour.

\section{Conclusions}

A novel approach to reduce the computation complexity of the wavelet transform has been presented in this paper. Two key techniques have been applied in this new method, namely, fast number theoretic transform (FNTT) and twodimensional overlap-save technique. In the fast number theoretic transform, the linear convolution is replaced by the circular convolution. It can speed up the computation of $2 \mathrm{D}$ discrete wavelet transform. Directly calculating the fast number theoretic transform to the whole input sequence may meet two difficulties; namely, a big modulo obstructs the effective implementation of the fast number theoretic transform and a long input sequence slows the computation 
of the fast number theoretic transform down. To fight with such deficiencies, a new technique which is referred to as $2 \mathrm{D}$ overlap-save method has been developed. Experiments have been conducted. The fast number theoretic transform and $2 \mathrm{D}$ overlap-method have been used to implement the dyadic wavelet transform and applied to contour extraction in image processing and pattern recognition.

\section{Conflict of Interests}

The authors declare that there is no conflict of interests regarding the publication of this paper.

\section{Acknowledgments}

This work was supported by the Research Grants MYRG205(Y1-L4)-FST11-TYY and MYRG187(Y1-L3)FST11-TYY and Chair Prof. Grants RDG009/FST-TYY of University of Macau, as well as Macau FDC Grants T-1002012-A3 and 026-2013-A. This research project was also supported by the National Natural Science Foundation of China 61273244.

\section{References}

[1] P. L. Combettes and J.-C. Pesquet, "Wavelet-constrained image restoration," International Journal of Wavelets, Multiresolution and Information Processing, vol. 2, no. 4, pp. 371-389, 2004.

[2] M. Ehler and K. Koch, "The construction of multiwavelet biframes and applications to variational image denoising," International Journal of Wavelets, Multiresolution and Information Processing, vol. 8, no. 3, pp. 431-455, 2010.

[3] P. Jain and S. N. Merchant, "Wavelet-based multiresolution histogram for fast image retrieval," International Journal of Wavelets, Multiresolution and Information Processing, vol. 2, no. 1, pp. 59-73, 2004.

[4] S. Mallat and W. L. Hwang, "Singularity detection and processing with wavelets," Institute of Electrical and Electronics Engineers: Transactions on Information Theory, vol. 38, no. 2, pp. 617-643, 1992.

[5] B. U. Shankar, S. K. Meher, and A. Ghosh, "Neuro-wavelet classifier for multispectral remote sensing images," International Journal of Wavelets, Multiresolution and Information Processing, vol. 5, no. 4, pp. 589-611, 2007.

[6] S. Shi, Y. Zhang, and Y. Hu, "A wavelet-based image edge detection and estimation method with adaptive scale selection," International Journal of Wavelets, Multiresolution and Information Processing, vol. 8, no. 3, pp. 385-405, 2010.

[7] Y. Y. Tang, Wavelets Theory Approach to Pattern Recognition, World Scientific, Singapor, 2009.

[8] Q. M. Tieng and W. Boles, "Wavelet-based affine invariant representation: a tool for recognizing planar objects in $3 \mathrm{D}$ space," IEEE Transactions on Pattern Analysis and Machine Intelligence, vol. 19, no. 8, pp. 921-925, 1997.

[9] P. Wunsch and A. F. Laine, "Wavelet descriptors for multiresolution recognition of handprinted characters," Pattern Recognition, vol. 28, no. 8, pp. 1237-1249, 1995.

[10] H. Yin and H. Liu, "A Bregman iterative regularization method for wavelet-based image deblurring," International Journal of
Wavelets, Multiresolution and Information Processing, vol. 8, no. 3, pp. 485-499, 2010.

[11] X. You and Y. Y. Tang, "Wavelet-based approach to character skeleton," IEEE Transactions on Image Processing, vol. 16, no. 5, pp. 1220-1231, 2007.

[12] T. Zhang, Q. Fan, and Q. Gao, "Wavelet characterization of Hardy space $h^{1}$ and its application in variational image decomposition," International Journal of Wavelets, Multiresolution and Information Processing, vol. 8, no. 1, pp. 71-87, 2010.

[13] Y. Y. Tang, L. Y. Li, Feng, and J. Liu, "Scale-independent wavelet algorithm for detecting step-structure edges," Tech. Rep., IEEE Transactions on Information Theory, Brisbane, Australia, 1998.

[14] S. G. Mallat, "Multiresolution approximations and wavelet orthonormal bases of $L^{2}(\mathbf{R})$," Transactions of the American Mathematical Society, vol. 315, no. 1, pp. 69-87, 1989.

[15] J. H. McClellan and C. M. Rader, Number Theory in Digital Signal Processing, Prentice Hall Signal Processing Series, Prentice Hall, Englewood Cliffs, NJ, USA, 1979.

[16] X. Ran and K. J. R. Liu, "Fast algorithms for 2-D circular convolutions and number theoretic transforms based on polynomial transforms over finite rings," IEEE Transactions on Signal Processing, vol. 43, no. 3, pp. 569-578, 1995.

[17] Q. Sun, "Some results in the application of the number theory to digital signal processing and public-key systems," in Number Theory and Its Applications in China, vol. 77 of Contemporary Mathematics, pp. 107-112, American Mathematical Society, Providence, RI, USA, 1988.

[18] Y. Y. Tang, Q. Sun, L. H. Yang, and L. Feng, "An overlapsave method for the calculation of the two-dimensional digital convolution," Tech. Rep., Department of Computer Science, Hong Kong Baptist University, 1998. 


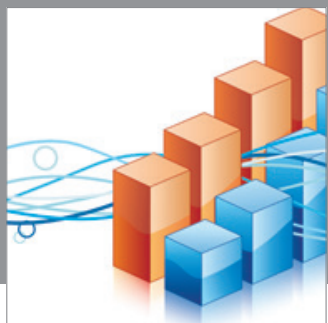

Advances in

Operations Research

mansans

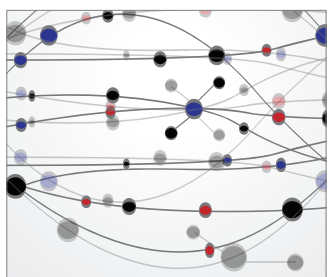

The Scientific World Journal
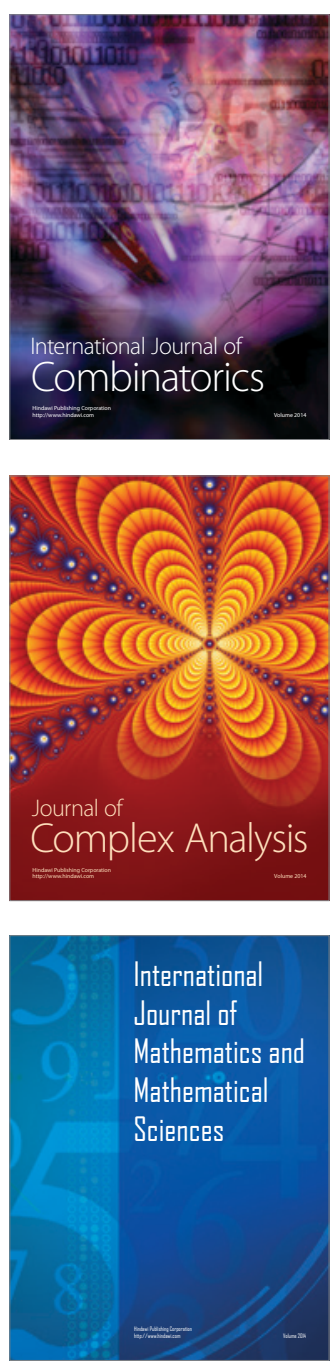
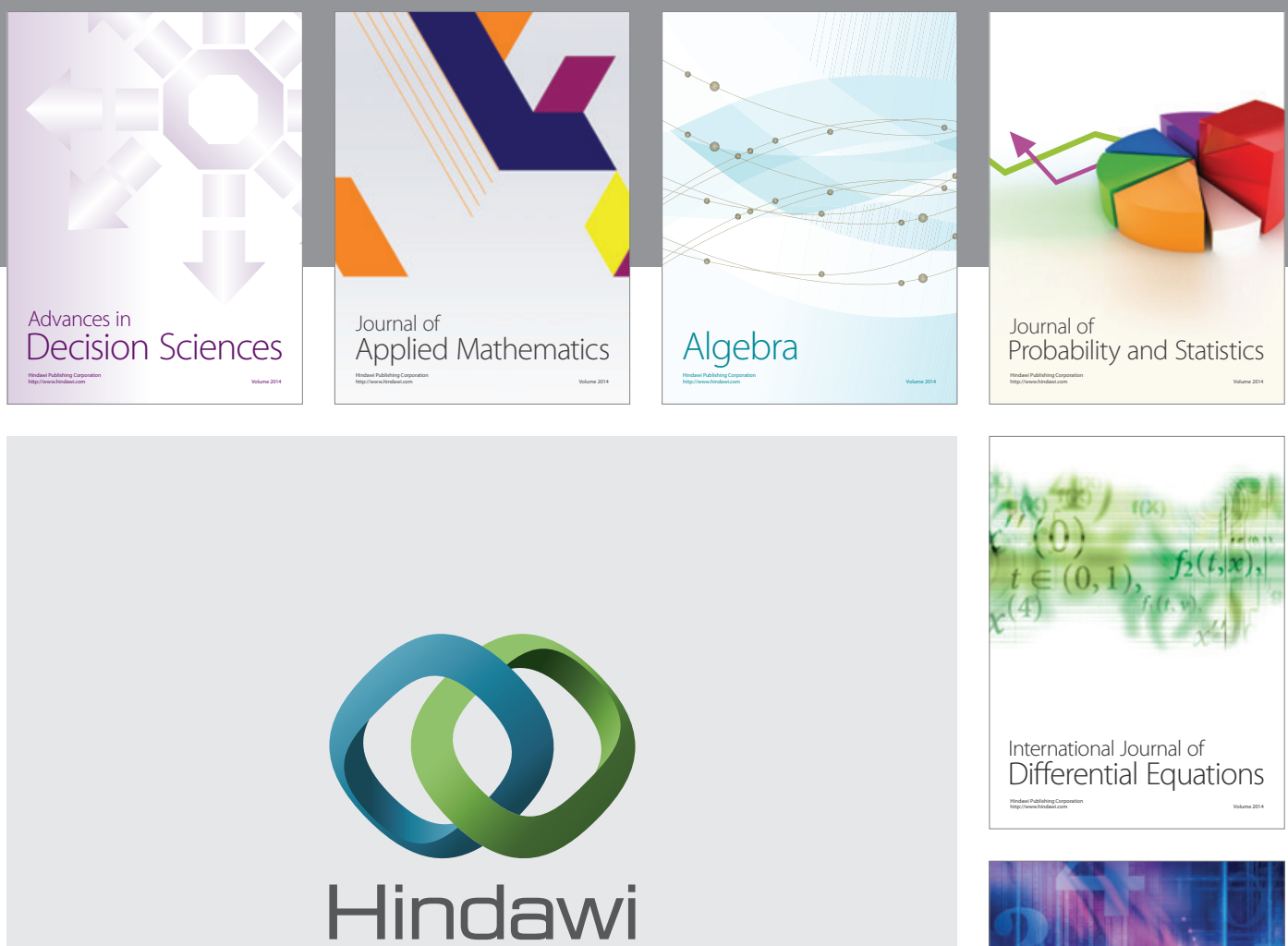

Submit your manuscripts at http://www.hindawi.com
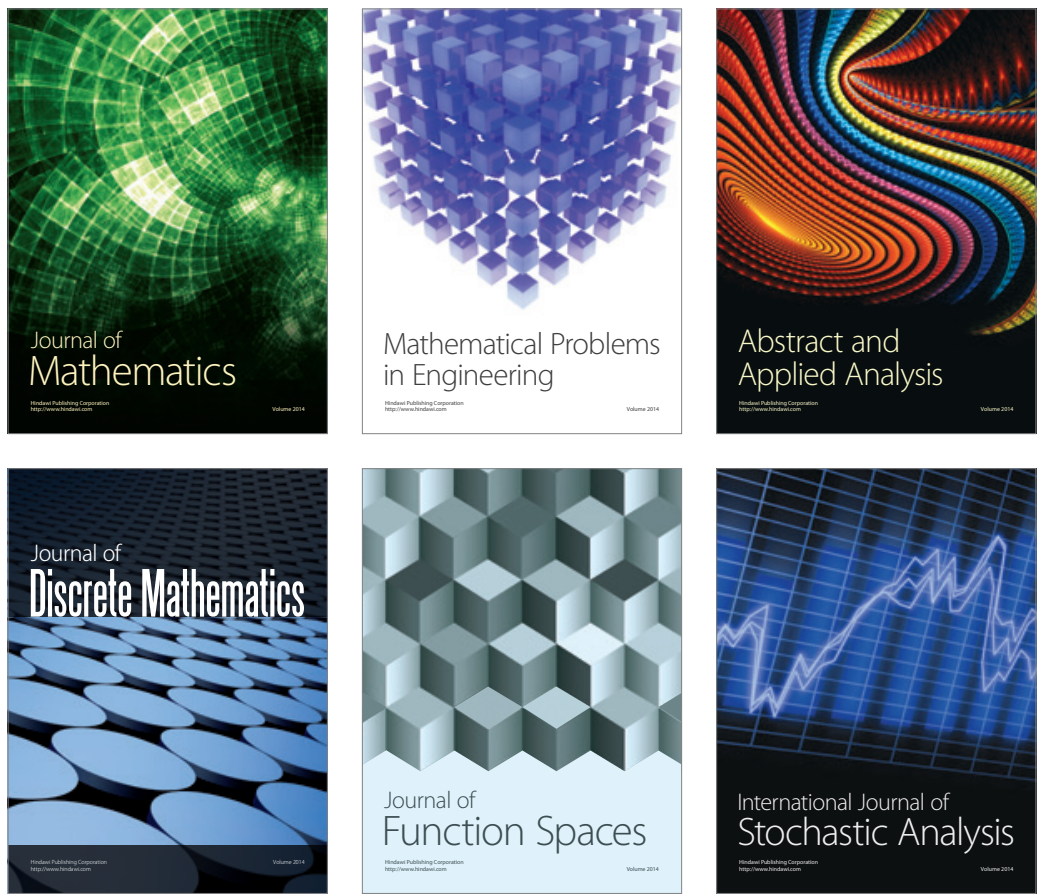

Journal of

Function Spaces

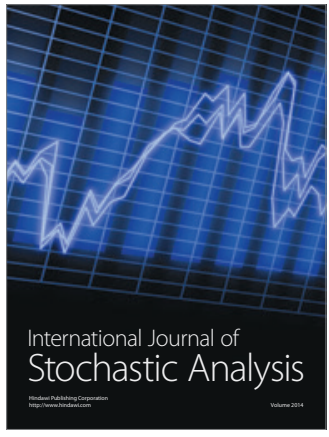

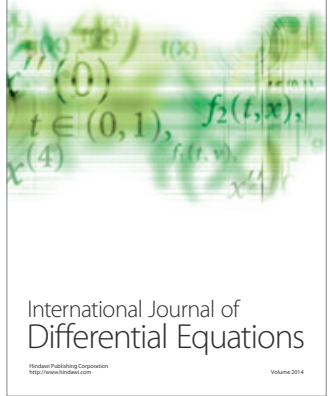
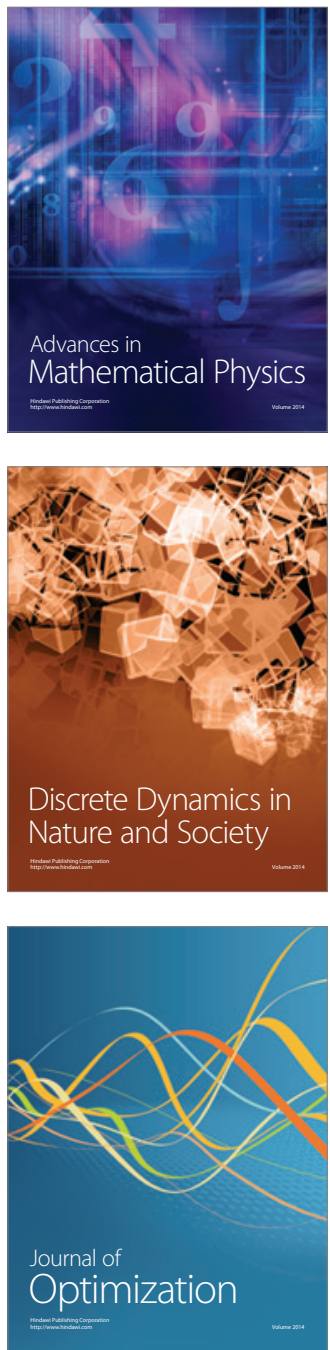